\title{
Using dust, gas and stellar mass-selected samples to probe dust sources and sinks in low-metallicity galaxies
}

\author{
P. De Vis, ${ }^{1,2 \star ~ H . ~ L . ~ G o m e z, ~}{ }^{3}$ S. P. Schofield, ${ }^{3}$ S. Maddox, ${ }^{3,4}$ L. Dunne,,${ }^{3,4}$ M. Baes, ${ }^{2}$ \\ P. Cigan, ${ }^{3}$ C. J. R. Clark, ${ }^{3}$ E. L. Gomez, ${ }^{5,3}$ M. Lara-López ${ }^{6}$ and M. Owers ${ }^{7,8}$ \\ ${ }^{1}$ Department of Physics and Astronomy, University of Canterbury, Private Bag 4800, Christchurch, New Zealand \\ ${ }^{2}$ Sterrenkundig Observatorium, Universiteit Gent, Krijgslaan 281, B-9000 Gent, Belgium \\ ${ }^{3}$ School of Physics and Astronomy, Cardiff University, Queen's Buildings, The Parade, Cardiff CF24 3AA, UK \\ ${ }^{4}$ Institute for Astronomy, University of Edinburgh, Royal Observatory, Blackford Hill, Edinburgh EH9 3HJ, UK \\ ${ }^{5}$ Las Cumbres Observatory Global Telescope Network, 6740 Cortona Dr, Suite 102, Goleta, CA 9117, USA \\ ${ }^{6}$ Instituto de Astronomía, Universidad Nacional Autómana de México, A.P. 70-264, 04510 México, D.F., Mexico \\ ${ }^{7}$ Australian Astronomical Observatory, P.O. Box 915, North Ryde, NSW 1670, Australia \\ ${ }^{8}$ Department of Physics and Astronomy, Macquarie University, NSW 2109, Australia
}

Accepted 2017 April 21. Received 2017 March 24; in original form 2016 November 30

\begin{abstract}
We combine samples of nearby galaxies with Herschel photometry selected on their dust, metal, $\mathrm{H}_{\mathrm{I}}$ and stellar mass content, and compare these to chemical evolution models in order to discriminate between different dust sources. In a companion paper, we used an $\mathrm{H}$ i-selected sample of nearby galaxies to reveal a subsample of very gas-rich (gas fraction $>80$ per cent) sources with dust masses significantly below predictions from simple chemical evolution models, and well below $M_{\mathrm{d}} / M_{*}$ and $M_{\mathrm{d}} / M_{\text {gas }}$ scaling relations seen in dust and stellar-selected samples of local galaxies. We use a chemical evolution model to explain these dust-poor, but gas-rich, sources as well as the observed star formation rates (SFRs) and dust-to-gas ratios. We find that (i) a delayed star formation history is required to model the observed SFRs; (ii) inflows and outflows are required to model the observed metallicities at low gas fractions; (iii) a reduced contribution of dust from supernovae ( $\mathrm{SNe}$ ) is needed to explain the dust-poor sources with high gas fractions. These dust-poor, low stellar mass galaxies require a typical core-collapse SN to produce $0.01-0.16 \mathrm{M}_{\odot}$ of dust. To match the observed dust masses at lower gas fractions, significant grain growth is required to counteract the reduced contribution from dust in $\mathrm{SNe}$ and dust destruction from SN shocks. These findings are statistically robust, though due to intrinsic scatter it is not always possible to find one single model that successfully describes all the data. We also show that the dust-to-metal ratio decreases towards lower metallicity.
\end{abstract}

Key words: dust, extinction-galaxies: dwarf-galaxies: evolution-galaxies: fundamental parameters - galaxies: ISM - galaxies: star formation.

\section{INTRODUCTION}

Interstellar dust is formed in the winds of evolved low-tointermediate-mass stars (LIMS, Ferrarotti \& Gail 2006; Sargent et al. 2010) and in core-collapse supernovae ( $\mathrm{SNe}$ ) as massive, short-lived stars end their lives (e.g. Dunne et al. 2003; Rho et al. 2008; Dunne et al. 2009; Barlow et al. 2010; Matsuura et al. 2011; Gomez et al. 2012; Indebetouw et al. 2014; Gall et al. 2014). There are also strong indications for grain growth in the interstellar medium (ISM) as dust grains acquire additional mass from the gas phase by accretion in both high- and low-redshift galaxies (Dwek, Galliano \& Jones 2007; Mattsson \& Andersen 2012; Asano et al. 2013; Zhukovska 2014; Rowlands et al. 2014b). Recent surveys with the Herschel Space Observatory (hereafter Herschel, Pilbratt et al., 2010) of nearby galaxies have produced dust mass scaling relations with stellar mass, gas mass and star formation rate (SFR) in both targeted samples of nearby galaxies, such as the Herschel Reference Survey (HRS, e.g. Boselli et al. 2010; Cortese et al. 2012; Smith et al. 2012; Cortese et al. 2014), and in wide-area blind surveys (Dunne et al. 2011; Bourne et al. 2012; Clark et al. 2015) including the Herschel-Astrophysical Terahertz Large area Survey (H-ATLAS, Eales et al. 2010). The dust properties of the blind, volume-limited, dust-selected Herschel-ATLAS Phase-1 LimitedExtent Spatial Survey (HAPLESS) sample of 42 galaxies over the 
Table 1. The average properties for the samples used in this work quoted as the mean \pm standard deviation. Where data are not available for all the sample, we quote the number of sources in the brackets. We only show the LTGs in the HRS

\begin{tabular}{llcccc}
\hline Galaxy sample & \multicolumn{1}{c}{$\begin{array}{c}\log \mathrm{SFR} \\
\left(\mathrm{M}_{\odot} \mathrm{yr}^{-1}\right)\end{array}$} & $\begin{array}{c}\log M_{\mathrm{HI}} \\
\left(\mathrm{M}_{\odot}\right)\end{array}$ & $\begin{array}{c}\log M_{*} \\
\left(\mathrm{M}_{\odot}\right)\end{array}$ & $\begin{array}{c}\log M_{\mathrm{d}} \\
\left(\mathrm{M}_{\odot}\right)\end{array}$ \\
\hline DGS & $-0.68 \pm 0.85(45)$ & $8.57 \pm 0.78(35)$ & $8.10 \pm 0.99$ & $5.12 \pm 1.77$ & $f_{\mathrm{g}}$ \\
HIGH-low & $-1.19 \pm 0.52$ & $9.02 \pm 0.46$ & $8.17 \pm 0.56$ & $5.21 \pm 0.97$ & $0.74 \pm 0.23(35)$ \\
HIGH-high & $-0.07 \pm 0.40$ & $9.76 \pm 0.39$ & $9.89 \pm 0.65$ & $7.12 \pm 0.43$ & $0.87 \pm 0.09$ \\
HRS (LTGs) & $-0.70 \pm 0.67$ & $8.94 \pm 0.56(231)$ & $9.64 \pm 0.57$ & $6.70 \pm 0.54(239)$ & $0.28 \pm 0.22(231)$ \\
\hline
\end{tabular}

equatorial H-ATLAS fields were presented by Clark et al. (2015, hereafter $\mathrm{C} 15$ ).

C15 attempted to model the HRS and HAPLESS galaxies using a simple closed box chemical evolution model and suggested the following: as galaxies evolve, their dust content first rises steeply, then levels off and reaches its peak about half-way through its evolution, and finally declines towards lower gas fractions. In De Vis et al. (2017, hereafter DV17), a local H I-selected sample taken from the same H-ATLAS fields (dubbed 'HIGH') was used to complement the stellar mass-selected HRS and the dust-selected HAPLESS sample. They showed that the $\mathrm{H}$ I selection recovered similar dust- and gas-rich galaxies as were seen in HAPLESS, but also revealed gasrich sources with much lower dust content. DV17 showed that these dust-poor sources are offset from the simple evolutionary scenario put forward in $\mathrm{C} 15$.

Zhukovska (2014) compared the sample from Rémy-Ruyer et al. (2014) (including the Dwarf Galaxy Survey (DGS, Madden et al. 2013), the largest sample of low-metallicity sources observed with Herschel) with a chemical evolution model to show that the observed variation in dust-to-gas ratio and metallicity in local SF dwarfs can be explained using models with bursty star formation histories (SFHs), low dust yields from core-collapse $\mathrm{SNe}$ and additional grain growth in the ISM. Feldmann (2015) took the sample from RémyRuyer et al. (2014) and used both an analytic approximation and dynamic one-zone chemical evolution models to fit the observed relationships in the 126 local galaxies. These models require very rapid grain growth, which activates at a critical metallicity, to match the observed dust-to-metal ratio in the galaxies. Feldmann (2015) also argues that there is a balance between metal-poor inflows and enriched outflows which regulates the dust-to-metal ratio. Popping, Somerville \& Galametz (2016) study the dust content of galaxies from $z=0$ to 9 using chemical evolution models including stellar dust, dust grain growth, destruction of dust by $\mathrm{SNe}$ and in the hot halo, and dusty winds and inflows.

In this companion paper to DV17, we add additional metallicity information to DV17's compilation of dust, stellar mass and $\mathrm{H}$ I-selected samples of nearby galaxies, and add the metalselected DGS, in order to investigate the dust-to-gas and dust-tometal properties for a total of 382 sources (44 DGS sources, 58 HAPLESS+HIGH sources and 280 HRS sources have dust masses from Herschel photometry). The combined sample here allows one to sample a wider range of gas fractions than possible before (from $\left.0.05<f_{\mathrm{g}}<0.97\right)$. We apply a chemical evolution model to interpret the data by relaxing the closed box model assumption from C15 and DV17 and adding inflows and outflows, using different SFHs, allowing for dust grain growth in the ISM and dust destruction. Section 2 reminds the reader of the samples from DV17 and introduces the DGS. Section 3 describes the new metallicity data and the used calibrations. Section 4 briefly describes the chemical model and the combination of parameters modelled in this work. The results are discussed in Section 5, where we attempt to deter- mine the contribution from different dust sources and to explain the dust-poor, gas-rich sample first shown in DV17. Our conclusions are listed in Section 6.

\section{NEARBY GALAXY SAMPLES}

A detailed description of the HRS, HAPLESS and HIGH samples used in this work is provided in detail in DV17. Here, we briefly remind the reader of the different data sets and parameters, introduce the new metallicity measurements and introduce the DGS (Madden et al. 2013; Rémy-Ruyer et al. 2013) which we add to our sample of local Herschel galaxies in Section 4. The average properties for the samples used in this work are shown in Table 1. By compiling the different nearby galaxy samples, we can model the dust properties for a total of 382 sources, compared to 126 sources in Zhukovska (2014) and Feldmann (2015). We also increase the number of low-metallicity sources (additional 67 sources with $Z<1 / 3 Z_{\odot}$ ), which lie off the scaling relations for more evolved sources. This is particularly important given the relevance of immature, unevolved low-metallicity sources as analogues for the first galaxies. When comparing all samples, $M_{\mathrm{d}} / M_{\text {bary }}$ first rises steeply, then levels off and then drops again as galaxies evolve from high to low gas fractions.

\subsection{H I, dust and stellar mass-selected samples}

The dust-selected HAPLESS (C15) is a blind, volume-limited sample of 42 local $(z<0.01)$ galaxies detected at $250 \mu \mathrm{m}$ from the H-ATLAS Phase 1 Version-3 internal data release, covering $160 \mathrm{deg}^{2}$ of the sky (Valiante et al. 2016; Bourne et al. 2016). The H I-selected sample (HIGH, DV17) is extracted from the same $\mathrm{H}$ ATLAS area and includes 40 unconfused $\mathrm{H}_{\text {I }}$ sources identified in the H H Parkes All Sky Survey (Barnes \& Hernquist 1992; Meyer et al. 2004) and the Arecibo Legacy Fast ALFA Survey (Giovanelli et al. 2005; Haynes et al. 2011, Haynes et al. private communication); 24 of these sources overlap with the HAPLESS sample. DV17 further split their sample by stellar mass into $\mathrm{HIGH}$-high and $\mathrm{HIGH}-$ low categories based on whether the sources were above or below $M_{*}=10^{9} \mathrm{M}_{\odot}$. To supplement the dust and $\mathrm{H}$ I-selected samples taken from H-ATLAS, we follow C15 and DV17 and use the HRS (Boselli et al. 2010) which provides a (quasi-) stellar massselected sample of nearby sources. The HRS targeted 323 galaxies ranging from late- to early-type sources. $\mathrm{H}_{\mathrm{I}}$ masses were taken from Boselli, Cortese \& Boquien (2014). DV17 compiled far-ultravioletsubmillimetre (FUV-submm) photometry for each of these samples, and subsequently derived dust masses, stellar masses and SFRs consistently using MAGPHYS (da Cunha, Charlot \& Elbaz 2008). The MAGPHYS properties for the HIGH and HAPLESS samples are provided in Table A1. 


\subsection{The Dwarf Galaxy Survey}

In this work, we also include results from the DGS (Madden et al., 2013) to improve our sampling of galaxies at low stellar masses and metallicities. The DGS sources were selected from several other surveys in order to make a broad sample of 50 galaxies ranging from very low $\left(\sim 1 / 50 Z_{\odot}\right)$ to moderate metallicity $\left(\sim 1 / 3 Z_{\odot}\right)$. In order to compare the samples, we need consistent methods to calculate the different galactic properties. Unfortunately, we do not have the same complete UV-submm coverage for DGS sources as we have available for the H-ATLAS and HRS. Consequently, we redetermined the dust masses for the DGS galaxies using a two-component modified blackbody (MBB) fit to the $70-500 \mu \mathrm{m}$ photometry provided in Rémy-Ruyer et al. (2013, 2015). This method produces consistent results with the dust masses output by MAGPHYS for the HAPLESS and $\mathrm{HIGH}$ sources with dust temperatures $>15 \mathrm{~K}$ and both methods assume the same dust absorption coefficient of $\kappa_{850}=0.07 \mathrm{~m}^{2} \mathrm{~kg}^{-1}$ (James et al. 2002). However, for some sources fitted by an MBB with $T_{\mathrm{c}}<15 \mathrm{~K}$, MAGPHYs results in warmer temperatures (by $\sim 3 \mathrm{~K}$ ) and therefore smaller dust masses (see also DV17). After scaling the Rémy-Ruyer et al. (2015) masses for graphite grains by the difference in $\kappa$ used in their work and MAGPHYS, these dust masses are entirely consistent with the MBB results for all sources with $T_{\mathrm{c}}>15 \mathrm{~K}$. For some of the colder sources there is an offset, yet this is of the same magnitude as the offset between MAGPHYS and the MBB method. Therefore in what follows, we simply scale the Rémy-Ruyer et al. (2015) dust masses for the difference in $\kappa$ to be consistent across samples.

Rémy-Ruyer et al. (2015) derived stellar masses based on the Eskew, Zaritsky \& Meidt (2012) method. To check consistency with the MAGPHYs stellar masses, we rederived stellar masses for our $\mathrm{HIGH}$ sample using their calibration and found that the DGS stellar masses were a factor of $\sim 3.2$ larger than MAGPHYs. This difference stems mainly from Eskew et al. (2012) adopting a Salpeter initial mass function (IMF), whereas in this work we assume a Chabrier IMF. After scaling the DGS values to be consistent with the other samples here, the HIGH-low and DGS contain galaxies with similar average stellar masses. SFRs for DGS were taken from RémyRuyer et al. (2015) and were estimated using a combination of $L_{\mathrm{TIR}}$ and the observed $\mathrm{H} \alpha$ luminosity (Kennicutt et al. 2009). As there are no integrated $\mathrm{H} \alpha$ luminosities available for the $\mathrm{HIGH}$ and HAPLESS samples, we compared this SFR method with the SFRs output by MAGPHYS for the HRS galaxies. We found that these methods were compatible for all but the most quiescent sources in the HRS (specific SFR $<10^{-11} \mathrm{M}_{\odot} \mathrm{yr}^{-1}$ ) which are not discussed here. Hi masses are available for 35 DGS galaxies from Madden et al. (2013).

\section{METALLICITIES AND CALIBRATIONS}

To calculate metallicities for the HIGH and HAPLESS samples, we use fibre optical spectroscopy from Sloan Digital Sky Survey (SDSS, Thomas et al. 2013), supplemented by the v17 Galaxy And Mass Assembly (GAMA) Survey (Hopkins et al. 2013). Although GAMA is an extragalactic survey of thousands of galaxies, we have used GAMA fibre spectra that, for our nearby galaxies, correspond to $\mathrm{H}$ II regions within the galaxies. Emission lines were measured by running each spectrum through a modified version of the Gas AND Absorption Line Fitting algorithm (GANDALF; Sarzi et al., 2006). Results were cross-checked with GAMA's GAUSSFITCOMPLEXV05 (GFC; Gordon et al., 2016) and both techniques gave comparable results for all but $15 \mathrm{H}$ II regions. The results for these $15 \mathrm{H}$ II regions were checked against the Fit3D (Sánchez et al. 2016) and the GAMA SpecLines (v4) catalogues (Hopkins et al. 2013) and found to be consistent with the GFC method.

For many of the HIGH and HAPLESS sources, we found multiple fibres within the same galaxy. SF (H II) regions were selected using the criteria in Kauffmann et al. (2003) by placing sources on the BPT diagram (Baldwin, Phillips \& Terlevich 1981). This resulted in a sample of $95 \mathrm{H}$ II regions for the $40 \mathrm{HIGH}$ galaxies and $85 \mathrm{H}$ II regions for the 42 HAPLESS galaxies $\left(67 \mathrm{H}_{\text {II }}\right.$ regions overlap as their galaxies are in both samples). The emission-line fluxes for each $\mathrm{H}$ II region were corrected for stellar absorption, and for internal and galactic extinction using the Balmer decrement $\mathrm{C}\left(\mathrm{H}_{\beta}\right)$ and the Cardelli, Clayton \& Mathis (1989) dust obscuration curve. Errors on the line measurements were provided by GANDALF or GFC. We then bootstrapped the measurements by generating 1000 new emission-line fluxes assuming a normal distribution with the extinction-corrected emission-line fluxes as mean and the measured error as the standard deviation of the distribution. The HIGH and HAPLESS emission lines and their errors are presented in Table A3. For the HRS, emission lines from integrated spectroscopy are available from Boselli et al. (2013) for 170 late-type galaxies (LTGs). DGS line measurements are taken from the literature (Table A4; Madden, private communication). ${ }^{1}$

To derive metallicities from the emission-line spectra, we compared the results from different empirical and theoretical methods in order to understand any systematic differences that may result from our methods. Here, our aim is to compare the different calibrations to determine which one fits our model best. Empirical calibrations are only valid for the same range of excitation and metallicity as the $\mathrm{H}$ II regions that were used to build the calibration. Since they are determined assuming an electron temperature, these methods may systematically underestimate the true metallicity if there are temperature inhomogeneities in a galaxy. This is thought to be more severe in metal-rich $\mathrm{H}$ II regions because the higher efficiency of metal-line cooling leads to stronger temperature gradients (Garnett 1992; Stasińska 2005; Moustakas et al. 2010). On the other hand, theoretical calibrations require inputs including stellar population synthesis and photoionization models; often the theoretical metallicities are higher than those found with the empirical calibrations.

We therefore chose to compare three common empirical methods including O3N2 and (third-order polynomial) N2 from Pettini \& Pagel (2004) and the S calibration from Pilyugin \& Grebel (2016, hereafter PG16S). All three methods produce metallicities that correlate well with stellar mass and gas fraction (e.g. Kewley \& Ellison 2008). However, the O3N2 calibration is only calibrated for metallicities $12+\log (\mathrm{O} / \mathrm{H})>8$ (Pettini \& Pagel 2004; Marino et al. 2013), and the $\mathrm{N} 2$ method also runs into difficulties at the lowest metallicities due to the large scatter observed in N/O ratios (Morales-Luis et al. 2014) and instead provides upper limits to the true metallicity for galaxies when $12+\log (\mathrm{O} / \mathrm{H})_{\mathrm{N} 2}<8$. We also derived metallicities consistent with the theoretical calculations from Tremonti et al. (2004, hereafter T04). As we do not have access to their code we used the scaling relation between O3N2-T04 from Kewley \& Ellison (2008), calibrated against 27,730 SDSS $\mathrm{SF}$ galaxies (hereafter KE08/T04). We note that this conversion is only valid for $8.05<12+\log (\mathrm{O} / \mathrm{H})_{\mathrm{O} 3 \mathrm{~N} 2}<8.9$. Additionally, we have determined metallicities using the Bayesian-based IZI tool (Blanc et al. 2015) which also provides a theoretical calibration

\footnotetext{
${ }^{1}$ We include the DGS emission lines and metallicities for the community in Table A4, and we correct some of the references.
} 
based on photoionization models. The IZI results were found to be entirely consistent with the KE08/T04 calibration and are thus not further listed in this work. When the different calibrations are compared, we find the PG16S calibration produces lower metallicities, followed by N2, O3N2 and then KE08/T04 (see also Table 3). Because the limited validity of the other calibrations below $12+$ $\log (\mathrm{O} / \mathrm{H})=8, \mathrm{PG} 16 \mathrm{~S}$ is the most reliable calibration for the lowmetallicity sources. Given the importance of low-metallicity sources in this work, we will plot metallicities using the PG16S calibration throughout. However, for high-metallicity sources, there is a remaining uncertainty in the metallicity relations in this work with respect to which calibration is used, and we therefore highlight the main differences throughout the text and include results derived from all three calibrations where appropriate.

Metallicities for each $\mathrm{HIGH}$ and HAPLESS galaxy were derived from a weighted average of the multiple $\mathrm{H}$ II regions within the same source (Table A2). Errors were derived by adding in quadrature the bootstrapped values on the extinction-corrected emission lines and the intrinsic scatter observed between the different $\mathrm{H}$ II regions within the same galaxy. The latter amounts to an uncertainty of 0.06 dex (see also Bresolin \& Kennicutt 2015). Note that these metallicities are based on an average of multiple small ( 2 " for GAMA and 3" for SDSS) fibres per source and not on integrated measurements. They are thus not ideally suited for extended sources, yet they at least provide a good first estimate for the metallicity of the galaxies in our sample. The calibrations used in this work are derived using the electron temperature method. The uncertainty in the absolute metallicity determination by this method is $\sim 0.1 \mathrm{dex}$ (Kewley \& Ellison 2008). We thus add 0.1 dex in quadrature to the uncertainty on the averaged metallicity for each galaxy and each calibration. The resulting metallicities and uncertainties for $\mathrm{HIGH}$ and HAPLESS are listed in Table A2.

For the HRS and DGS, we simply derive metallicities based on the integrated spectroscopy for each of the three calibrations. The DGS metallicities were originally estimated using the Pilyugin \& Thuan (2005, hereafter PT05) calibration (Madden et al. 2013). PT05 is calibrated over a similar range of metallicity to N2 and PG16S, but there are a number of reasons we choose not to use this as a method to determine metallicities in this study. First, PT05 is not a good estimator for metal-rich galaxies that have low excitation parameters $P$ and high values of $R_{23}$ (Moustakas et al. 2010), such as the HRS and HiGH-high galaxies. PT05 is therefore more suited for the DGS and HIGH-low sources that have appropriate $P$ and $R_{23}$ values, but this means we cannot apply a consistent method to derive metallicities across the different samples of nearby galaxies. Second, PT05 metallicities have been shown to have a lot of scatter with stellar mass compared to other calibrators (Kewley \& Ellison 2008), suggesting it is not a good tracer of metallicity across a wide range of galaxy properties. Third, the PT05 method has two 'branches' of metallicity values versus the $R_{23}$ emission-line ratio with a transition region between the two branches. Because of this, a large difference in $Z$ can be derived for galaxies with very small changes in emission-line ratio. The PG16S calibration also uses different relations for high and low metallicities. However the appropriate ranges where the high- and low-metallicity relations can be used, overlap for adjacent metallicities, and the transition zone thus disappears. Fig. 1 compares the DGS metallicities derived here with the published values from Madden et al. (2013, M13), which are based on the PT05 calibration. We find, on average, lower metallicities for galaxies with $12+\log (\mathrm{O} / \mathrm{H})_{\mathrm{M} 13}<8$, and higher metallicities for high-metallicity sources (though with less scatter). We see that the differences between PT05 and PG16S are less pronounced than

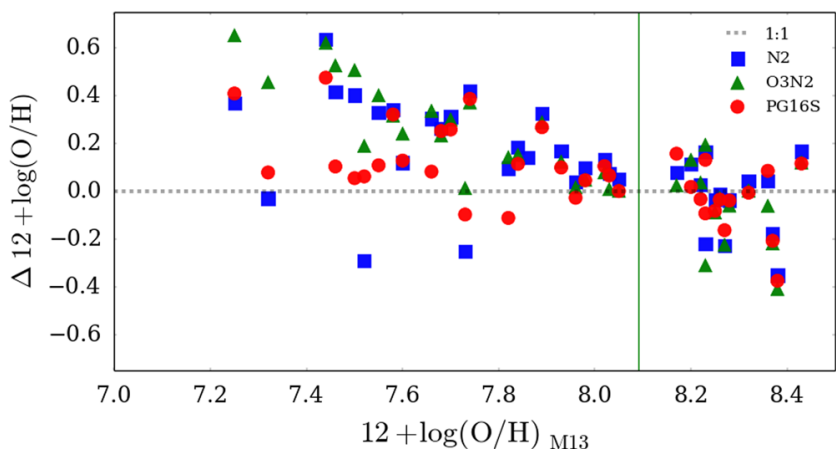

Figure 1. A comparison of the differences in metallicity calibrations $\Delta(12+\log (\mathrm{O} / \mathrm{H}))$ using the $\mathrm{N} 2$ (blue), O3N2 (green) and PG16S (red) methods with the published DGS metallicities derived using PT05 (M13, Madden et al. 2013). The significant offset between the O3N2 and PT05 at the lowest metallicities may be because this calibration is known to breakdown here (indicated by the vertical line; Pettini \& Pagel 2004), though the N2 and PG16S results also suggest PT05 tends to underestimate metallicities in this regime.

for the other calibrations, though using PG16S still suggest higher metallicities ( 0.09 dex on average) than previously published, especially for low-metallicity sources. We thus use PG16S metallicities throughout this work.

\section{THE CHEMICAL EVOLUTION MODEL}

A chemical and dust evolution model can be used to build a consistent picture of how the metals, dust and gas content change as galaxies evolve (Tinsley 1980). The simple chemical evolution model used in $\mathrm{C} 15$ to interpret the different scaling relations for dust, gas and stellar mass-selected samples neglected dust destruction and grain growth, and assumed that the system was a closed box (no inflows or outflows). Following Zhukovska (2014) and Feldmann (2015), we relax all of these assumptions. The chemical model is presented in full in Rowlands et al. (2014b; see also Morgan \& Edmunds, 2003) and the PYTHON code used is freely available on GITHUB. ${ }^{2}$ The equation for the dust mass evolution is given in Appendix B. In short, the model uses a prescription for the SFH and a Chabrier (2003) IMF to calculate how much of the initial gas is converted into stars at any given time. The model also tracks the continuous build-up of metals as stars end their lives, though metals can be removed in outflows of material. For dust, the picture is more complex. Dust is produced by SNe and evolved LIMS, and additional mass is gained from the ISM by dust grain growth. Dust is primarily destroyed by SN shocks and astration (the removal of gas and dust due to SF). We use a one-zone chemical evolution model, i.e. we study the integrated properties of galaxies without spatial resolution, and assume instant mixing of dust, gas and metals.

In this model, we include simple analytical prescriptions for grain growth and dust destruction via shocks as described in Rowlands et al. (2014b). The time-scale for dust destruction ( $\tau_{\text {dest }}$, following Dwek et al. 2007) is described as a function of the rate of $\mathrm{SN}\left(R_{\mathrm{SN}}\right)$ :

$\tau_{\mathrm{dest}}=\frac{M_{\mathrm{g}}}{m_{\mathrm{ISM}} R_{\mathrm{SN}}(t)}$,

where $M_{\mathrm{g}}$ is the gas mass of the galaxy and $m_{\mathrm{ISM}}$ is the mass of ISM that is swept up by each individual SN event. In some models (Table 2), we set this to $m_{\mathrm{ISM}}=100 \mathrm{M}_{\odot}$, indicative of $\mathrm{SN}$ shocks

${ }^{2}$ https://github.com/zemogle/chemevol 
Table 2. Parameters for the different chemical evolution models used.

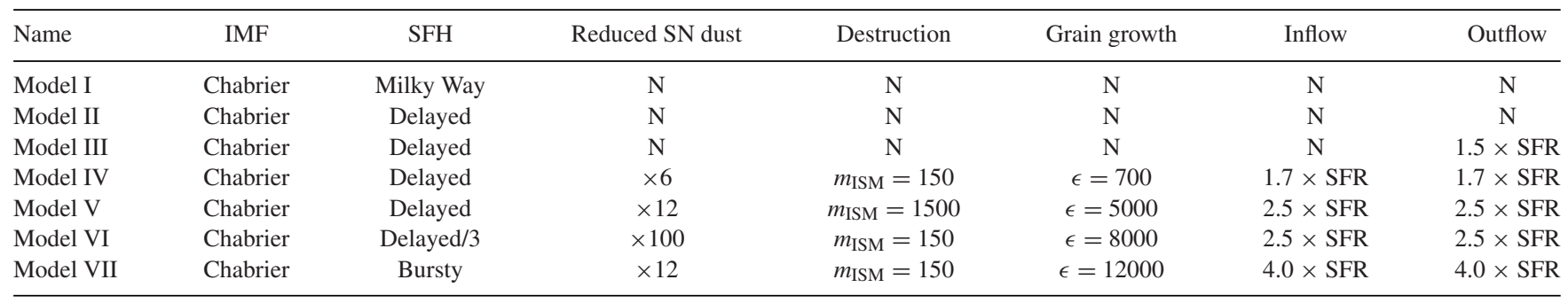

ploughing into typical interstellar densities of $10^{3} \mathrm{~cm}^{-3}$ (Gall, Andersen \& Hjorth 2011; Dwek \& Cherchneff 2011), although we also explore models with $1000 \mathrm{M}_{\odot}$ (Dwek et al. 2007), consistent with dust destruction in the diffuse ISM and possibly more appropriate for a low-metallicity ISM.

The grain growth prescription is taken from Mattsson \& Andersen (2012), where the time-scale for dust growth is given by:

$\tau_{\text {grow }}=\frac{M_{\mathrm{g}}}{\epsilon Z \psi(t)}\left(1-\frac{\eta_{\mathrm{d}}}{Z}\right)^{-1}$,

and $\eta_{\mathrm{d}}$ is the dust-to-gas ratio, $Z$ is the metallicity $\left(Z=M_{\text {metals }} / M_{\text {gas }}\right)$ and $\epsilon$ is a free parameter, which is set to $\epsilon=500$ in Mattsson \& Andersen (2012), appropriate for accretion time-scales of $10^{7} \mathrm{yr}$ for a galaxy similar to the Milky Way (MW).

We also test four 'representative' SFHs, including a MW-type exponentially declining SFR (Yin et al. 2009), and two versions of a delayed SFH as parametrized by Lee et al. (2010):

$\operatorname{SFR}(t) \propto \frac{t}{\tau^{2}} e^{-t / \tau}$

where $t$ is the age of the galaxy and $\tau$ is the SF time-scale. First, we assume a SFH with $\tau=6.9 \mathrm{Gyr}$ with peak SFR $4.4 \mathrm{M}_{\odot} \mathrm{yr}^{-1}$ in order to produce the same stellar mass as the MW-type SFH. The second delayed SFH is reduced by a factor of 3, and has $\tau=15 \mathrm{Gyr}$ (see Section 5.3). Finally, a model including a bursty SFH, similar to that used in Zhukovska (2014) to explain the SFR properties of the DGS sources, is also included. In Section 5, we test various parameter combinations, including changing SFHs, IMFs, inflows, outflows and including different dust sources, in order to interpret the observed dust, metal, gas and SFRs of the nearby galaxy samples. The parameters for Models I-VII, which are good representations for the sampled parameter space, are listed in Table 2.

We note that this model differs from Rowlands et al. (2014b) in the following ways: (i) the initial remnant mass function is updated. (ii) We now take into account the formation of a black hole for stars with initial mass $m_{\mathrm{i}}>40 \mathrm{M}_{\odot}$ when accounting for gas and metals released into the ISM. Stars with progenitor mass above this cut-off mass only contribute gas and metals lost via stellar winds before the collapse. (iii) We add an additional term $f_{\mathrm{c}}$ to account for the fraction of gas that is cold enough for grain growth in the ISM. We follow Mancini et al. (2015) and Inoue (2003) by setting this equal to 0.5 . This parameter is likely to be higher at earlier times (e.g. Popping, Somerville \& Trager 2014; Nozawa et al. 2015) though we choose to keep it constant here. (iv) We no longer interpolate the yields from stars of a given mass but just choose the nearest neighbour value, this has a small effect on the resulting stellar yields. (v) We directly input the dust masses for core-collapse SN for stars with initial mass $8.5<M_{\mathrm{i}} \leq 40$ from Todini \& Ferrara (2001). Rowlands et al. (2014b) used the Todini \& Ferrara (2001) dust masses to estimate a condensation efficiency for SN dust $\left(\delta_{\mathrm{SN}}\right)$ and applied that to the metal yields from Maeder (1992). Using the

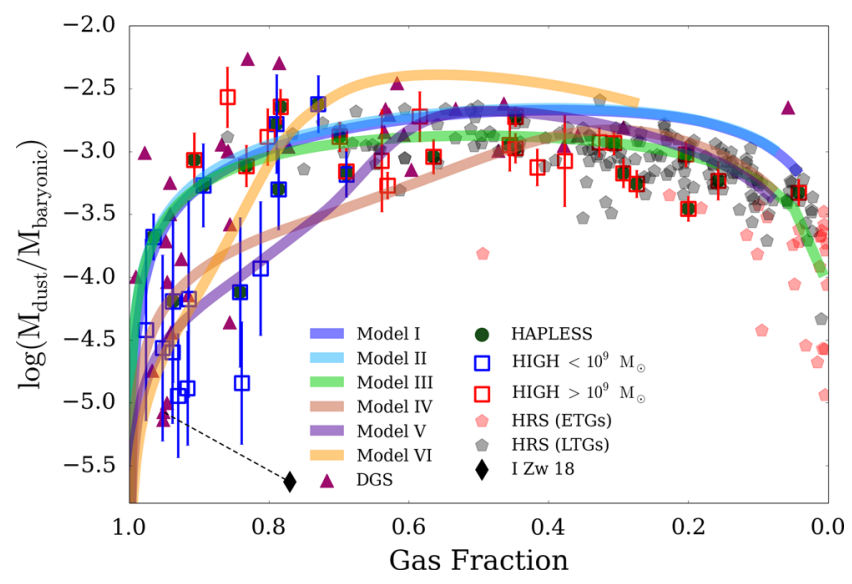

Figure 2. Variation of $M_{\mathrm{d}} / M_{\text {bary }}$ with gas fraction for the different nearby galaxy samples. The solid lines show how galaxies with the same initial gas mass, but different combinations of SFHs, inflows, outflows and dust sources evolve as the gas is consumed into stars (Models I-VI, Table 2). Models I and II overlap in this plot. The observed properties of dust-poor local galaxy I Zw 18 (black diamond) are also added for comparison (Fisher et al. 2014), with dashed line indicating where this source 'moves' using the methods in this work.

former technique reduces the dust mass by a factor of $\sim 1.8$ for an MW-like galaxy at early times $(<0.8 \mathrm{Gyr})$ compared to the latter.

\section{RESULTS}

\subsection{A simple model fit to dust in nearby galaxies}

In Fig. 2, we follow C15 and DV17 and compare the evolution of the dust-to-baryonic mass ratio $\left(M_{\mathrm{d}} / M_{\text {bary }}\right)$ with gas fraction for the different nearby galaxy samples compiled here. This plot is an excellent starting point as it tracks the relative changes in dust mass in terms of the evolutionary state. We define the baryon mass and gas fraction as $M_{\text {bary }}=M_{\mathrm{g}}+M_{*}$ and $f_{\mathrm{g}}=\frac{M_{\mathrm{g}}}{M_{*}+M_{\mathrm{g}}}$ respectively, where $M_{\mathrm{g}}=1.32 M_{\mathrm{HI}}$ to take into account the mass of neutral helium. Due to the difficulty in obtaining CO detections for all the different samples considered here, particularly for low stellar mass sources, we do not take into account any molecular component. We refer to Section 5.9 for further discussion, though we note here that $\mathrm{H}_{2}$ does not dominate the total gas mass for our samples and thus including $\mathrm{H}_{2}$ would not affect the conclusions reached in this work.

In Fig. 2, we find $M_{\mathrm{d}} / M_{\text {bary }}$ follows a tight relation at low gas fractions. However at high gas fraction there is more scatter, at least in part due to differences in the contributions from the different dust sources. We also show how the observations from the different samples compare with a chemical evolution track similar to $\mathrm{C} 15$ and DV17. This model uses an SFH consistent with the MW (Yin et al. 2009), though here we use our updated code (Model I, 
Table 2). Model I overlaps with Model II in Fig. 2 (see also Section 5.2). Although galaxies are more complex than this simple model, Model I does explain the overall trend in these samples, yet not all sources at gas fractions $<50$ per cent are well matched. We note that our model peaks at a lower gas fraction $(\sim 0.3)$ than in C15 and DV17 due to the changes made to the assumptions and dust inputs described in Section 4. Indeed, as our model has less dust injection from $\mathrm{SNe}$ but the same dust injection from LIMS compared to Rowlands et al. (2014b), this shifts the peak $M_{\mathrm{d}} / M_{\text {bary }}$ towards lower gas fractions. In this work, we assume a dust condensation efficiency for LIMS of 0.16, consistent with predictions of Morgan \& Edmunds (2003). This value is somewhat lower than the high condensation efficiencies from theoretical models of dust formation in stellar winds (Zhukovska, Gail \& Trieloff 2008; Ventura et al. 2012). By choosing an even lower value for the dust condensation efficiency in LIMS, we could obtain a better fit to $M_{\mathrm{d}} / M_{\text {bary }}$ at low gas fractions for the closed box model of $\mathrm{C} 15$. However, as we will show in Section 5.2, an equally good fit to $M_{\mathrm{d}} / M_{\text {bary }}$ can be obtained through the introduction of inflows and outflows when we relax the assumptions of the closed box model.

In Fig. 2, we see that a large fraction of the highest gas-fraction galaxies $\left(f_{\mathrm{g}}>85\right.$ per cent, HiGH-low) have significantly lower $M_{\mathrm{d}} / M_{\text {bary }}$ than expected from Model I. We note, however, that the dust mass of these galaxies have large error bars due to poorly constrained dust temperatures from the MAGPHYs fitting. In order to ensure the offset in $M_{\mathrm{d}} / M_{\text {bary }}$ for these sources is not due to this, we stacked the mid-infrared-submm fluxes for the $8 \mathrm{HIGH}$-low sources with poorly constrained temperatures. The resulting stacked SED is well fitted by a single MBB curve with dust temperature $T \sim 35 \mathrm{~K}$. The lower dust masses for these sources are therefore consistent with them having warmer dust temperatures than the HAPLESS and HRS sources (on average). We conclude that a different set of chemical model properties are necessary to explain this slower build-up of dust for these high gas-fraction sources compared to the dust-rich HAPLESS galaxies at the same $M_{\mathrm{d}} / M_{\text {bary }}$.

Note that in Fig. 2 we also highlight the well-studied galaxy I Zw 18 (part of the DGS sample) thought to be a local analogue of lowmetallicity, high-redshift systems (e.g. Herrera-Camus et al. 2012; Fisher et al. 2014). The location of this source on this $M_{\mathrm{d}} / M_{\text {bary }}$ 'scaling relation' (and in later sections) is indicated by the black diamond using the measured properties from Fisher et al. (2014). As we have re-evaluated the DGS measurements to be consistent across all samples (Section 2), we have indicated where this galaxy moves with our revised measurements (dashed line). We will see in later sections that the dust properties of I $\mathrm{Zw} 18$ are entirely consistent with its gas fraction and metallicity.

\subsection{Relaxing the closed box assumption}

Fig. 2 also compares the $M_{\mathrm{d}} / M_{\text {bary }}$ of these samples with different chemical evolution tracks including different SFHs and/or relaxing the closed box assumption from Model I (Models II-VI, Table 2). There are significant differences between some of the models and the data, especially at $f_{\mathrm{g}} \sim 80$ per cent. Even for the same gas fraction, nearby low $M_{*}$ galaxies split into two categories: dustrich and dust-poor and require different chemical evolution models to explain their dust-to-baryonic mass properties. Briefly, we see that the dust-rich low $M_{*}$ sources are matched relatively well by Models I-III, which show a steep rise in $M_{\mathrm{d}} / M_{\text {bary }}$ at the highest gas fractions $\left(f_{\mathrm{g}}>95\right.$ per cent) and correspond to core-collapse $\mathrm{SNe}$ producing $0.17-1.0 \mathrm{M}_{\odot}$ of dust per explosion when there is no dust destruction or grain growth (or the net interstellar grain growth is matched by equal dust destruction). For the sources with high $M_{\mathrm{d}} / M_{\text {bary }}$ for their gas fraction, an increased SN dust yield results in a better fit. In this case, the dust contribution from LIMS needs to be reduced. Even though the SFH for Models I and II are very different, their chemical evolution tracks in Fig. 2 nearly overlap, indicating the $M_{\mathrm{d}} / M_{\text {bary }}$ evolution is not dependent on the SFH for models without dust grain growth.

To model the dust-poor HiGH-low and DGS sources, we follow Zhukovska (2014) and Feldmann (2015) and relax the closed box assumption, reduce the contribution from SN dust, and include dust grain growth in our model. Models IV-VI therefore require a reduction in the dust production in SNe by a factor of 6-100 compared to the models required to fit the HRS, HIGH-high and HAPLESS. In contrast to our approach (and Zhukovska, 2014), Feldmann (2015) even uses reduced SN dust yields for sources that are not dust-poor given their metallicity and instead uses extremely fast (time-scale of 5 Myr) grain growth to achieve high dust masses at high gas fractions. There is thus a degeneracy between using a significant contribution from SN dust, and using very fast dust grain growth. Their grain growth time-scales of $\sim 5 \mathrm{Myr}$ are much faster than typically found in nearby galaxies (Mattsson \& Andersen 2012; Mattsson et al. 2014) or from basic theoretical estimates of the underlying growth rate (Draine 2009).

At late times (low gas fractions), Models I and II overestimate the amount of $M_{\mathrm{d}} / M_{\text {bary }}$ and require (stronger) inflows and dustrich outflows of gas or a reduced dust contribution from LIMS to explain the observed properties. The choice of Models I-VI will be motivated in Sections 5.3-5.6.

\subsection{Star formation histories}

Next we attempt to explain the observed SFR properties with these models by comparing the change in SFR/ $M_{\text {bary }}$ with gas fraction. Fig. 3 compares the HAPLESS, HRS, HIGHand DGS samples. In the high gas-fraction regime $\left(f_{\mathrm{g}}>80\right.$ per cent $)$, we see that Model I overpredicts the SFR $/ M_{\text {bary }}$, particularly in comparison to the HiGH-low sources. Delayed SFH models provide a closer match to this sample (as used in Models II-VI) by reducing the SFR per unit baryonic mass at early evolutionary stages. The values of the delayed SFHs in equation (3) were chosen to match the data in

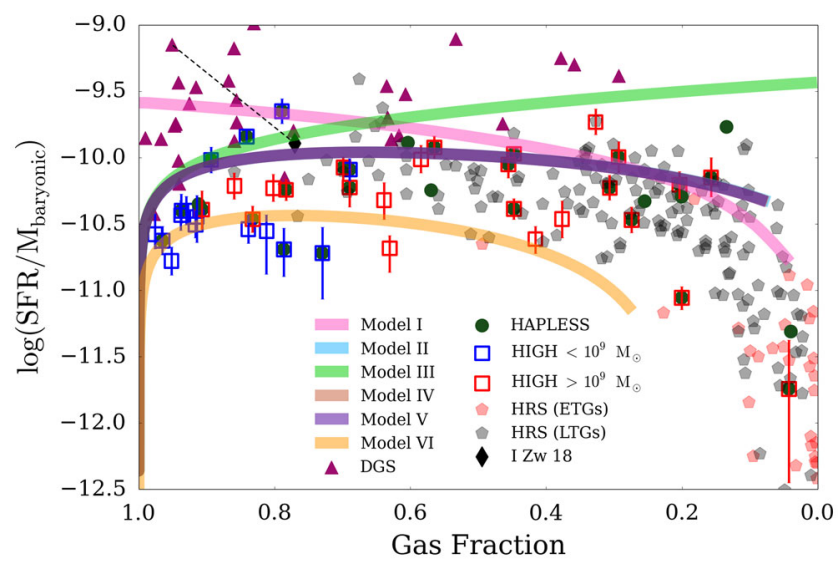

Figure 3. SFR $/ M_{\text {bary }}$ against the gas fraction reveals the need for a delayed SFH (Models II-VI) to explain the HIGH-low and HAPLESS sources at high $f_{\mathrm{g}}$. In this parameter space, Models II, IV and V overlap as they have the same SFH and their inflows and outflows are balanced. Bursty SFHs are needed for the DGS (Appendix C). 
Fig. 3, with Model VI providing a good fit to most ${ }^{3}$ of the HIGHlow sources. In models with strong outflows but no inflows (Model III), the baryonic mass is significantly reduced at low gas fractions, and therefore SFR $/ M_{\text {bary }}$ increases as the gas fraction decreases. Model III thus poorly matches the observed SFR $/ M_{\text {bary }}$ at low gas fractions and can be discarded as an unrealistic model. However, when the outflow is matched by an equal inflow as in Model V, $M_{\text {bary }}$ stays constant and we find the same SFR/ $M_{\text {bary }}$ track as for the same model without inflows and outflows (i.e. Models II, IV and $\mathrm{V}$ overlap in Fig. 3).

The DGS sources lie significantly above the HRS, HIGH and HAPLESS samples in Fig. 3, with higher SFR/ $M_{\text {bary }}$ for the same gas fraction. DGS tends to contain more actively SF galaxies (average SFR $0.21 \mathrm{M}_{\odot} \mathrm{yr}^{-1}$, Table 1) than is typical of nearby dwarfs (e.g. Hunter et al. 2012). Their selection towards more SF, lowstellar mass systems could be a consequence of their original selection of galaxies with moderate to very low PT05 metallicities. We return to this in the next section. The intensely SF nature of the DGS was highlighted in Zhukovska (2014), where they found they required bursty SFRs to fit the gas and dust properties of these dwarf galaxies. Even with the revised dust masses and metallicities and the different model assumptions in this work, we also require a bursty SFH to fit the DGS properties (Appendix C). This demonstrates that despite having similar stellar masses, dust temperatures and gas fractions as the HIGH-low sources, the DGS are more actively SF than the HIGH galaxies and do not appear to be the same sources at a different evolutionary stage. However, we cannot rule out that DGS and HiGH-low are both part of the same evolutionary sequence, with DGS sources undergoing a burst and HIGH-low sources in a quiescent period between bursts. The HiGH-low and HAPLESS samples therefore complement the DGS and provide additional, new, information of more normal SF systems at low metallicities, high $f_{\mathrm{g}}$ and potentially different dust properties.

\subsection{Metallicity build-up}

We next wish to compare how the metallicity of galaxies changes as they evolve from high to low gas fractions. The chemical evolution code traces both the total metal mass fraction $Z$ and the oxygen mass, we can directly convert the models to oxygen abundance using:

$$
12+\log \left(\frac{\mathrm{O}}{\mathrm{H}}\right)=12+\log \left(\frac{\text { oxygen mass } / 16}{\text { gas mass } / 1.32}\right) .
$$

In Fig. 4, we see in both the model behaviour and the observations that, in general, the metallicity increases monotonically as galaxies evolve from high to low gas fractions. The models are almost indistinguishable at gas fractions $>80$ per cent in this parameter space. At low gas fractions, Models I and II clearly overestimate the amount of metals; we find models with moderate $(2.5 \times$ SFR $)$ outflows of enriched gas and metal-poor inflows are necessary (Models V and VI). Here, we note that it is possible that empirical calibrations such as PG16S are underestimated (particularly at low gas fractions) due to temperature inhomogeneities. If this is the case, a theoretical calibration such as KE08/T04 would be more applicable and less strong inflows and outflows would be sufficient.

Fig. 4 also shows that the DGS appears to have lower metallicities than the HRS at low gas fractions and, to a lesser extent the

\footnotetext{
${ }^{3}$ Three HIGH-low sources actually have a higher SFR, more in line with the actively SF DGS sources rather than the other normal SF HIGH galaxies.
}

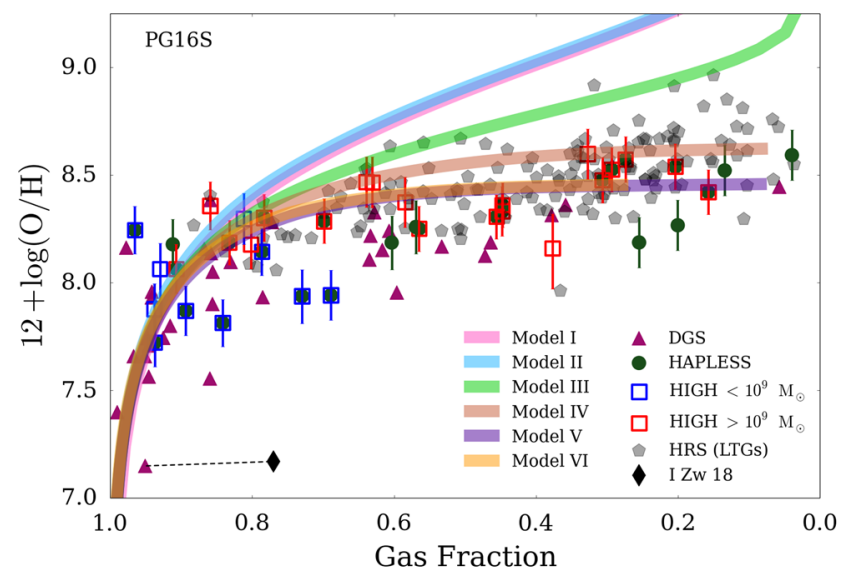

Figure 4. Metallicity variation with gas fraction for the different samples using the PG16S metallicity calibration. The different chemical evolution models (see the text and Table 2) are also included.

HiGH-low sources at high gas fractions. In other words, the DGS galaxies are, on average, more metal-poor given their evolutionary state. In the previous section, we also found DGS are, on average, more actively forming stars. Selecting galaxies ranging from low to moderate metallicity at a given gas fraction appears to result in a sample selection biased towards galaxies with very high SFRs due to the mass-metallicity-SFR relation (Mannucci et al. 2010; Lara-López et al. 2010). Additionally, higher SFR (and thus brighter) sources are easier to observe with Herschel, and it is easier to obtain high signal-to-noise spectra (in order to determine metallicities). This is another reason DGS consists mainly of high SFR sources. As suggested in Feldmann (2015), the low metallicities at a given gas fraction for DGS sources, requires the addition of strong inflows and outflows to regulate the build-up of metals in the DGS galaxies. Only Model VII (Fig. C1), with bursty SFH and stronger inflows/outflows $(4 \times$ SFR) than the models $(\mathrm{V}$ and VI) used to match the other nearby galaxy samples in this work, can be used to explain the $Z-f_{\mathrm{g}}$ properties of the DGS sample.

\subsection{Dust-to-gas ratio}

Next we compare metallicity with the dust-to-gas ratio for the 253 galaxies in our combined sample that have metallicities available (Fig. 5). The $M_{\mathrm{d}} / M_{\mathrm{g}}$ ratio correlates with the gas-phase metallicity over a wide range $7.3<12+\log (\mathrm{O} / \mathrm{H})_{\mathrm{PG} 16 \mathrm{~S}}<9.0$, yet we again identify two regimes. If dust traces the metals or a constant fraction of metals remains in dust grains, we expect a linear $M_{\mathrm{d}} / M_{\mathrm{g}}-Z$ relationship, with a slope as for Models I-III. Models I-III are consistent with the slope of those galaxies with highest $M_{\mathrm{d}} / M_{\mathrm{g}}$ in the metallicity range $7.3<12+\log (\mathrm{O} / \mathrm{H})_{\mathrm{PG} 16 \mathrm{~S}}<8.2$, but the HRS sources do lie offset from these models. ${ }^{4}$ This could be explained either by increasing the dust produced by stars by a factor of 5 or more which would move Models I-III up the $y$-axis whilst keeping the slope constant. However, we note that the amount of dust formed in LIMS stars and SNe is already substantial, and these models have no dust destruction suggesting that adding more dust produced from stars in this way is unrealistic. Alternatively, one could add interstellar grain growth which would act to steepen the tips of Models I-III at metallicites $12+\log (\mathrm{O} / \mathrm{H})_{\mathrm{PG} 16 \mathrm{~S}}>8.2$ (see, for

\footnotetext{
${ }^{4}$ Here, we do not aim to find a single model that explains the HRS galaxies, rather we simply show how models without grain growth evolve in this plot.
} 


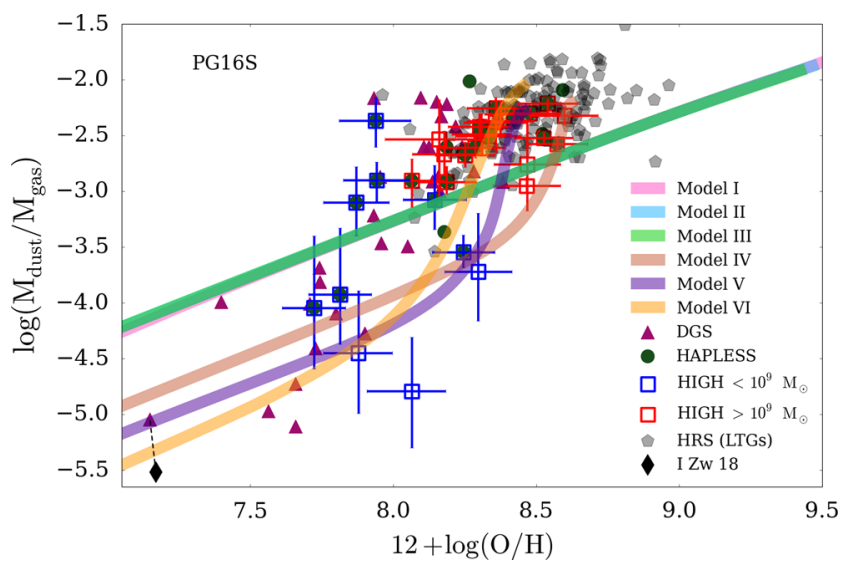

Figure 5. Metallicity variation with gas-to-dust ratio $M_{\mathrm{d}} / M_{\mathrm{g}}$. Models IVVI provide a better match between metallicity and $M_{\mathrm{d}} / M_{\mathrm{g}}$ for the HIGH-low and many DGS sources than Models I-III.

example the tips of Models IV-VI). We return to how this may be a result of different dust-to-metal ratios in Section 5.6.

The dust-poor high gas-fraction sources (HIGH-low and some of the DGS galaxies) lie well below the linear trend from Models I-III. This offset was already discussed in Rémy-Ruyer et al. (2013, 2015), Zhukovska (2014) and Feldmann (2015), who explained this by suggesting the SN contribution to the dust budget needs be reduced and a dust grain growth term added. The argument is such: for the highest gas-fraction galaxies in Fig. 5, the dust mass needs to be significantly suppressed without reducing the metals. The only way ${ }^{5}$ to do this is to reduce the amount of dust formed by stars (including $\mathrm{SN}$ ) in each stellar population. As the dustto-gas ratio is already lower than expected from a linear trend at high gas fractions, this suggests the $\mathrm{SN}$ dust production must be suppressed. The observed dust-to-gas ratio in the lowest metallicity HIGH-low and DGS galaxies requires models with a maximum of $0.01-0.16 \mathrm{M}_{\odot}$ (Models VI-IV) of dust per core-collapse SNe, which corresponds to a condensation efficiency of 0.2-3.2 per cent for a $25 \mathrm{M}_{\odot}$ progenitor (assuming $5 \mathrm{M}_{\odot}$ of metals ejected and that all of this mass can be condensed into dust).

Therefore Models IV-VI include a reduced SN dust component (by a factor of 6-100 in mass, Table 2) compared to the MW model. Since there is less stardust in these models, if we require galaxies to ultimately reach the typical dust-to-gas ratios observed at low $f_{\mathrm{g}}$ (Fig. 5), we need to also include interstellar grain growth. This dust source is strongly metal-dependent and only becomes important once the galaxy reaches a critical metallicity (Asano et al. 2013). This means that different values of the grain growth parameters $\epsilon$, and consequently $\tau_{\text {grow }}$, move the model tracks. An increase of $\epsilon$ steepens the slope of $M_{\mathrm{d}} / M_{\mathrm{g}}$ (shown by Models IV-VI as they reach the end of their tracks); any offset from the linear trend in Fig. 5 can therefore be mitigated by changing $\epsilon$ such that grain growth starts at a lower metallicity (thereby increasing the dust-to-gas ratio). Alternatively, offsets in Fig. 5 can also be explained through the use of different bursty SFHs, because long quiescent phases allow accretion of existing metals after short active enrichment episodes (Zhukovska 2014).

The relative contributions to the dust mass budget for Models IV-VI are displayed in Fig. 6. At high gas fractions, stellar sources dominate (mostly SN dust, Rowlands et al. 2014b, C15), yet dust

\footnotetext{
${ }^{5}$ Note the dust destruction caveat in Section 5.9.
}
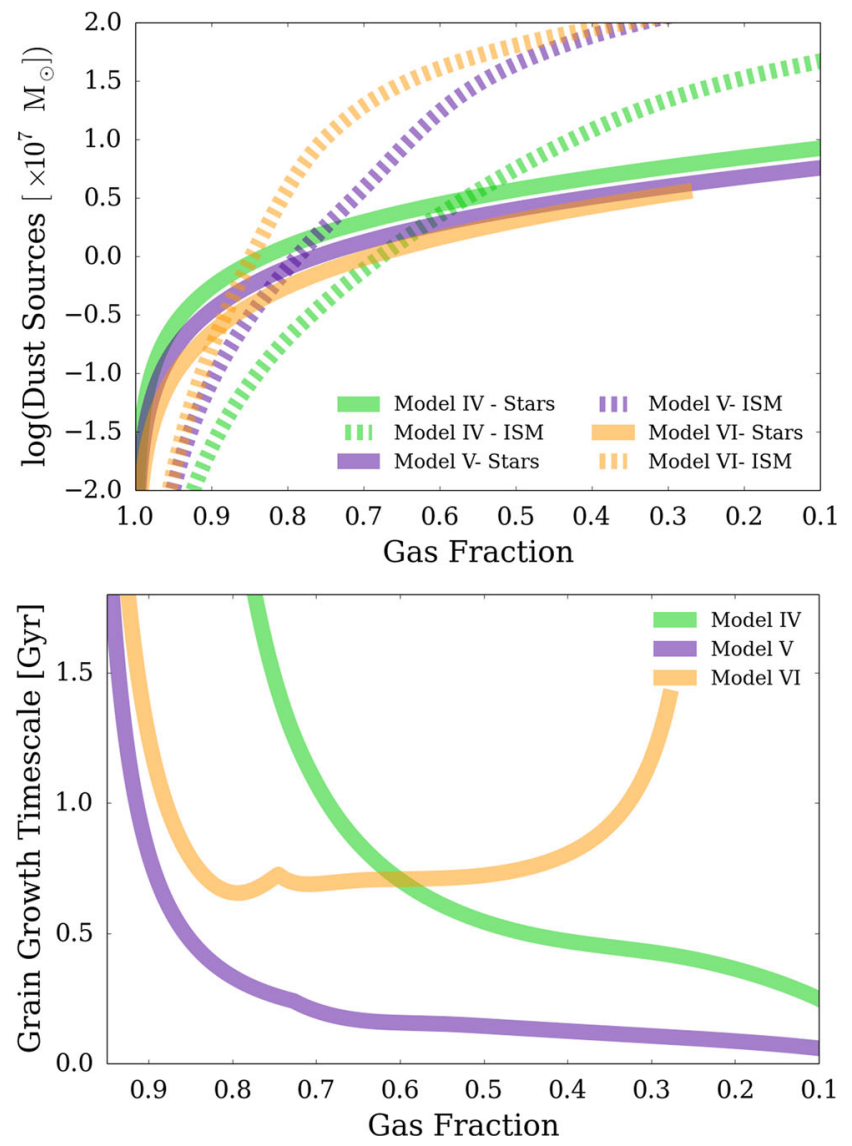

Figure 6. Top: the dust mass produced by the various dust sources in Models IV-VI against gas fraction. Stellar dust sources dominate at the highest gas fractions and are overtaken by dust grain growth at lower gas fractions. Bottom: the variation of the grain growth time-scale $\tau_{\text {grow }}$ (equation 2 ) with gas fraction for Models IV-VI. The growth time-scale remains long until the critical metallicity is reached.

grain growth becomes the largest source of dust mass at gas fraction below $0.88,0.79$ and 0.53 for Model VI, V and IV, respectively. The metallicity at which dust grain growth exceeds dust production from stars in our model is reached between $0.003<Z<0.012$ (or $7.97<12+\log (\mathrm{O} / \mathrm{H})<8.63$, or $\left.0.88>f_{\mathrm{g}}>0.53\right)$, though low values in this range result in the best match with observations. High values of $\epsilon$ (Table 2) lead to short grain growth time-scales and low critical metallicities. By the time Models IV-VI reach the lowest gas fractions, dust grain growth produced 70-93 per cent of the total dust mass created over the galaxy's lifetime.

The (Z-dependent) dust grain growth time-scales for Models IV, $\mathrm{V}$ and VI are shown in Fig. 6 (bottom). For Model IV and V, the dust grain growth time-scale $\tau_{\text {grow }}$ decreases steeply at high gas fractions (when the critical metallicity is reached), and decreases gradually after that. For Model VI, we again find an initial steep decrease in $\tau_{\text {grow }}$ at high gas fractions, yet afterwards we find some fluctuation and then an increase towards the lowest gas fractions. $\tau_{\text {grow }}$ is inversely proportional to the SFR (equation 2) and the Model VI SFR decreases steeply towards low gas fractions (and is low in general). This explains the higher $\tau_{\text {grow }}$ of Model VI compared to Model V, even though $\epsilon$ is higher. The fluctuation in the $\tau_{\text {grow }}$ for Model VI is thus a result of the balance of the growing efficiency of grain growth as the metallicity increases and the SFH reaching a peak and decreasing steeply towards low gas fractions. We note that 


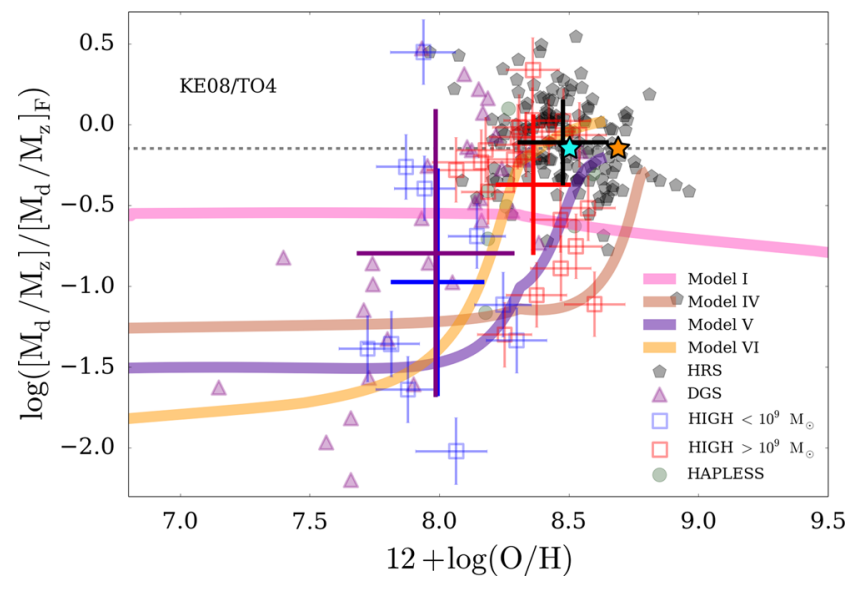

Figure 7. Dust-to-metal ratio versus metallicity (to allow comparison with Feldmann (2015), we use a normalization ${ }^{6}$ of $\left[M_{\mathrm{d}} / M_{\mathrm{Z}}\right]_{\mathrm{F}}=0.7$ ) for $\mathrm{HIGH}$, HRS, HAPLESS and DGS. The dust-to-metal ratio is significantly lower for galaxies in the low-metallicity regime regardless of how actively SF these galaxies are. The large crosses show the mean \pm standard deviation of dust-to-gas within the samples. We also highlight the $\mathrm{MW}\left(M_{\mathrm{d}} / M_{\mathrm{Z}}=0.5\right.$, orange star) and recent estimates for galaxies in the Virgo Cluster (Davies et al. 2014, cyan star).

even though the dust grain growth is slower in Model VI than Model $\mathrm{V}$, it is still faster relative to the SFR and thus a more important term in the chemical evolution.

\subsection{Dust-to-metal ratio}

The variation in the dust-to-metals ratio is discussed in Mattsson et al. (2014), where they show there is only a small change in $M_{\mathrm{d}} / M_{\mathrm{Z}}$ (but increased scatter) observed in low-metallicity environments (De Cia et al. 2013) and at high redshifts (Zafar \& Watson 2013) even down to $Z<1$ per cent of solar. In Fig. 7, we find that for HRS, and a small fraction of the other sources, there is indeed not much variation in the $M_{\mathrm{d}} / M_{\mathrm{Z}}{ }^{7}$. The HRS is in good agreement with the MW value (Clark et al. 2016) and the recent survey by Davies et al. (2014) using Herschel observations of galaxies in the Virgo Cluster. Models with stardust only (Model I) predict an almost constant dust-to-metals ratio. ${ }^{8}$

However, there are also $\sim 25$ low-metallicity (DGS and $\mathrm{HIGH}$ ) sources for which $M_{\mathrm{d}} / M_{\mathrm{Z}}$ is significantly smaller. We note this result is independent on which metallicity calibration was used. Results for N2 and T04/KE08 are included in Table 3 for comparison with PG16S. We thus use our larger and more normal SF sample at low $Z$ to further support the Feldmann (2015) result that the dust-to-metal ratio varies as a function of metallicity. The location of the low stellar mass samples (HIGH-low and DGS galaxies) is contrary to what we would expect if stellar sources were the dominant source of dust in the galaxies, which again shows we cannot model these sources without grain growth. Feldmann (2015) attributes the rising dust-to-metal ratio to dust grain growth becoming more efficient

\footnotetext{
${ }^{6}$ The normalization in Feldmann (2015) is given by their model $M_{\mathrm{d}} / M_{\mathrm{Z}}$ at solar metallicity.

${ }^{7}$ To estimate the total metal mass $M_{\mathrm{Z}}$ from the observed oxygen abundance from Section 3, we assume $12+\log (\mathrm{O} / \mathrm{H}) \odot=8.69$ and a solar metal mass fraction $\mathrm{Z}_{\odot}=0.014$ following Asplund et al. (2009).

${ }^{8}$ The kink in Model I at $12+\log (\mathrm{O} / \mathrm{H}) \sim 8.4$ is due to an increasing metal mass from stars resulting from changing the input metal yield file (Maeder 1992).
}

as galaxies reach their critical metallicity. In contrast to the strong inflows and outflows, and the extremely efficient interstellar grain growth (time-scale of $\sim 4 \mathrm{Myr}$ ) from Feldmann (2015), we find we can also model these sources with more moderate inflows and outflows $(2.5 \times \mathrm{SFR})$, and moderate grain growth (time-scale of 1 Gyr-200 Myr) models (Models IV-VI). The dust growth timescales in our models are more similar to those quoted for the MW and local galaxies (Draine 2009; Asano et al. 2013; Mattsson \& Andersen 2012; Mattsson et al. 2014).

We note it is very difficult for a model with SFH consistent with the MW, with dust from LIMS combined with significant dust production in SNe and no dust destruction or grain growth (Model I, peak $M_{\mathrm{d}} / M_{\mathrm{Z}} \sim 0.2$ ) to reach the observed MW dust-to-metals ratio $(\sim 0.5$; orange star in Fig. 7$)$ as it evolves. This issue demonstrates why significant interstellar grain growth is needed to supplement the dust mass and reduce the large offset in the predicted $M_{\mathrm{d}} / M_{\mathrm{Z}}$ in Model I compared to the observed values, even in our own Galaxy.

\subsection{Statistical constraints}

We next attempt to check whether the comparison between the data and Models I-VI in earlier sections provide strong constraints on the physical properties of these galaxies given the degeneracies in the model. The scatter in the observed values between the different galaxy samples compiled in this work and others, and indeed within samples are often much larger than their error bars. This suggests there is an intrinsic source of scatter in the observed data, and in this case, one would not expect one model to provide a good fit to the whole sample or even to subsets based on simple flux selection criteria. Here then, we focus on comparing whether one model, or class of models can provide a better description of the data than others, rather than derive the model that describes the data best in an absolute way. We aim to constrain the degeneracies and parameters of our chemical evolution models in a future paper (De Vis et al. in preparation ) using a Bayesian approach on a large grid of models. In this section, we will test whether the 'eye-ball fits' used here to select 'preferred models' (and in similar other studies Zhukovska 2014; Rémy-Ruyer et al. 2013; Feldmann 2015) are, in fact, statistically robust.

We do this by calculating a statistic to measure the goodness of fit between the data and each of the models as plotted in Figs 2, 3 and 5. For simplicity, we consider just two data samples: the full sample of all the galaxies together; and the HIGH-low subset. Using all of the galaxies equally weighted together is naturally dominated by the HRS sample which contains the most sources. The HIGHlow subset departs from the typical chemical trends seen in other, more evolved, galaxy samples, and so although we have to contend with small number statistics, this subset represents an unusual and interesting population.

Since our measurements have significant uncertainties in both the $x$ - and $y$-values, and our models are non-linear in $y$ as a function of $x$, it is not possible to simply use a standard $\chi^{2}$ approach to determine the goodness of fit. We use a Bayesian approach to determine an effective $\chi^{2}$ which includes the measurement uncertainties in two dimensions. We start from Bayes theorem:

$P($ model $\mid$ data $)=P($ data $\mid$ model $) \times P($ model $) / P($ data $)$.

For two measured parameters, $x$ and $y$, an observed data point $d_{\mathrm{i}}$ is given by $\left(x_{\mathrm{i}}, y_{\mathrm{i}}\right)$ with Gaussian uncertainties $\left(\sigma_{x_{\mathrm{i}}}, \sigma_{y_{\mathrm{i}}}\right)$. If we have a model linking $x$ and $y, y=f(x)$, and we know that the true value for $x$ is $x_{0}$, then the true value for $y$ is given by $f\left(x_{0}\right)$. For this situation, 
Table 3. Average dust-to-metal ratio for the different galaxy samples and metallicity calibrations quoted as mean \pm standard deviation.

\begin{tabular}{|c|c|c|c|c|c|c|}
\hline \multirow[t]{2}{*}{ Galaxy sample } & \multicolumn{3}{|c|}{ Mean $12+\log (\mathrm{O} / \mathrm{H})$} & \multicolumn{3}{|c|}{ Mean $M_{\mathrm{d}} / M_{\mathrm{Z}}$} \\
\hline & $\mathrm{N} 2$ & KE08/T04 & PG16S & N2 & KE08/T04 & PG16S \\
\hline DGS & $8.02 \pm 0.28$ & $8.34 \pm 0.19$ & $7.98 \pm 0.30$ & $-0.72 \pm 0.71$ & $-0.44 \pm 0.33$ & $-0.69 \pm 0.66$ \\
\hline HIGH-high & $8.48 \pm 0.12$ & $8.81 \pm 0.16$ & $8.36 \pm 0.14$ & $-0.49 \pm 0.43$ & $-0.82 \pm 0.42$ & $-0.37 \pm 0.43$ \\
\hline HRS (LTGs) & $8.57 \pm 0.15$ & $8.75 \pm 0.17$ & $8.48 \pm 0.18$ & $-0.20 \pm 0.24$ & $-0.38 \pm 0.25$ & $-0.11 \pm 0.27$ \\
\hline
\end{tabular}

the probability of observing $x_{\mathrm{i}}$ and $y_{\mathrm{i}}$ is given by:

$P\left(d_{\mathrm{i}} \mid\left(x_{0} \& f\right)\right) \propto \exp \left(-\frac{\left(x_{\mathrm{i}}-x_{0}\right)^{2}}{2 \sigma_{x_{\mathrm{i}}}^{2}}-\frac{\left(y_{\mathrm{i}}-f\left(x_{0}\right)\right)^{2}}{2 \sigma_{y_{\mathrm{i}}}^{2}}\right)$.

Since the MAGPHYS uncertainties used in this work are often asymmetric, we have used the lower $x$ error bar if $x_{0}<x_{i}$ and the upper error bar if $x_{0}>x_{\mathrm{i}}$, and equivalently for the $y$ error bars and $y_{\mathrm{i}}$ compared to $f\left(x_{0}\right)$.

To find the probability of observing the data point given the model $f$, we need to integrate over all possible values for the true value $x_{0}$. Assuming a uniform prior on $x_{0}$, this becomes,

$$
P\left(d_{\mathrm{i}} \mid f\right) \propto \int \exp \left(-\frac{\left(x_{\mathrm{i}}-x_{0}\right)^{2}}{2 \sigma_{x_{\mathrm{i}}}^{2}}-\frac{\left(y_{\mathrm{i}}-f\left(x_{0}\right)\right)^{2}}{2 \sigma_{y_{\mathrm{i}}}^{2}}\right) \mathrm{d} x_{0} .
$$

This integral can be solved numerically since the function $f\left(x_{0}\right)$ can be described numerically from the output of the chemical evolution models. The normalization for equation (7) is found from determining the maximum probability that would be obtained for any $y$-value for the same $x$-value as the real data point. We thus find the $y_{\max }$ that maximizes equation (7) with respect to $y$, and use this as the normalization:

$$
P\left(d_{\mathrm{i}} \mid f\right)=\frac{\int \exp \left(-\frac{\left(x_{\mathrm{i}}-x_{0}\right)^{2}}{2 \sigma_{x_{\mathrm{i}}}^{2}}-\frac{\left(y_{\mathrm{i}}-f\left(x_{0}\right)\right)^{2}}{2 \sigma_{y_{\mathrm{i}}}^{2}}\right) \mathrm{d} x_{0}}{\int \exp \left(-\frac{\left(x_{\mathrm{i}}-x_{0}\right)^{2}}{2 \sigma_{x_{\mathrm{i}}}^{2}}-\frac{\left(y_{\max }-f\left(x_{0}\right)\right)^{2}}{2 \sigma_{y_{\mathrm{i}}}^{2}}\right) \mathrm{d} x_{0}} .
$$

For a linear model, $y_{\max }=f\left(x_{0}\right)$, so a data point that lies on the model will correspond to $P\left(d_{\mathrm{i}} \mid f\right)=1$, which is equivalent to $\chi^{2}=$ 0 , as expected. This normalization reproduces the standard form of $\chi^{2}$ for a linear function with uncertainties in both $x$ and $y$. However, our models are not linear and as a result $y_{\max }$ is not necessarily equal to $f\left(x_{0}\right)$. By normalizing $P\left(d_{\mathrm{i}} \mid f\right)$ in this way, we ensure the estimated probability is never larger than 1 . This normalization is thus necessary to obtain sensible results. Formally we should normalize by the maximum of $P\left(d_{\mathrm{i}} \mid f\right)$ in both $x$ and $y$, but this $2 \mathrm{D}$ maximization is time-consuming, and our approximation is valid so long as $\sigma_{x} \frac{\mathrm{d}^{2} y}{\mathrm{~d} x^{2}}<\frac{\mathrm{d} y}{\mathrm{~d} x}$ and $\sigma_{y} \frac{\mathrm{d}^{2} x}{\mathrm{~d} y^{2}}<\frac{\mathrm{d} x}{\mathrm{~d} y}$, which is the case for our models and data.

The likelihood for the entire sample $\mathbf{d}=\left\{d_{\mathrm{i}}\right\}$ is then found from taking the product of each of the probabilities for the individual data points from equation (8). Equivalently, the logarithms of the probabilities can be added to give the log likelihood:

$$
\log (\mathcal{L}(\mathbf{d} \mid f))=\sum_{\mathrm{i}} \log \left(P\left(d_{\mathrm{i}} \mid f\right)\right) .
$$

Additionally, in order to allow for more easily interpretable results, we convert $P\left(d_{\mathrm{i}} \mid f\right)$ from equation (8) to an effective $\chi^{2}$ :

$\chi_{\mathrm{eff}}^{2}=\sum_{\mathrm{i}}-2 \log \left(P\left(d_{\mathrm{i}} \mid f\right)\right)$
Finally, so we can easily compare samples with different number of galaxies, $n$, we calculate the average contribution to $\chi_{\mathrm{eff}}^{2}$ per data point,

$$
\left\langle\chi_{\text {eff }}^{2}\right\rangle=\frac{\sum_{\mathrm{i}}-2 \log \left(P\left(d_{\mathrm{i}} \mid f\right)\right)}{n}
$$

This statistic is similar to reduced $\chi^{2}$, but does not allow for the number of degrees of freedom; the true number of degrees of freedom is hard to quantify because of correlations between the effects of parameters in our models. Nevertheless, our models are essentially different parameter choices for one overarching model, and so they all have the same effective degrees of freedom, and the statistic does allow a fair comparison between them.

In this section, we revisit three of the main figures in this work and determine how well Models I-VI fit the observations. Table 4 shows the $\left\langle\chi_{\text {eff }}^{2}\right\rangle$ for the combined HRS+HAPLESS $+\mathrm{HIGH}$ sample and for HiGH-low for Figs 2, 3 and 5. In these figures, the models do not reach the lowest gas fractions and highest metallicities. Therefore in our determination of $\left\langle\chi_{\text {eff }}^{2}\right\rangle$, we have discarded all data points with $f_{\mathrm{g}}<0.2$ for Figs 2 and 3 and all data points with $12+\log (\mathrm{O} / \mathrm{H})>$ 8.5 for Fig. 5. Figs 2 and 3 use gas fraction $f_{\mathrm{g}}$ on the $x$-axis. However, the uncertainties on $f_{\mathrm{g}}$ become very non-linear near $f_{\mathrm{g}} \sim 1$, so the approximation of a Gaussian error probability distribution is not valid, and the calculation of $\left\langle\chi_{\mathrm{eff}}^{2}\right\rangle$ is unreliable if we use $f_{\mathrm{g}}$ as the $x$ variable. Therefore, we have used $M_{\mathrm{g}} / M_{*}$ instead. This is entirely equivalent to $f_{\mathrm{g}}\left(M_{\mathrm{g}} / M_{*}=f_{\mathrm{g}} /\left(1-f_{\mathrm{g}}\right)\right)$, but has errors that are more close to Gaussian, and so our statistic is acceptable. It is worth noting that Models II and V provide a reasonable fit to each of the studied relations and for both subsamples $\left(\left\langle\chi_{\text {eff }}^{2}\right\rangle<4\right)$. In the rest of this section, we will study which of the models fits which relation best and how we interpret these results.

In Table 4 , we find that the observations for $M_{\mathrm{d}} / M_{\text {bary }}$ versus $M_{\mathrm{g}} / M_{*}$ (or equivalently $f_{\mathrm{g}}$ ) are best fitted by Model VI for $\mathrm{HIGH}$ low. We thus find statistical confirmation for our result from Section 5.2 that rapid dust grain growth is necessary to model the unevolved HiGH-low sources. For the combined sample, Model III provides the best fit. Here, the HRS dominates the $\left\langle\chi_{\text {eff }}^{2}\right\rangle$ because of its large sample size. As previously mentioned in Section 5.2, a reduced dust contribution from LIMS would reduce the $M_{\mathrm{d}} / M_{\text {bary }}$ at low $f_{\mathrm{g}}$ and thus provide a better match for Models I and II.

The large $\left\langle\chi_{\text {eff }}^{2}\right\rangle$ in Table 4 for SFR $/ M_{\text {bary }}$ against $M_{\mathrm{g}} / M_{*}$ for all models and both subsamples indicate that a single model cannot describe the spread in SFR $/ M_{\text {bary }}$. For both the combined sample and $\mathrm{HIGH}-$ low, Model I provides a very poor fit. Delayed SFH thus provide a better description of the overall SFH of normal SF galaxies than exponentially declining SFR. For the combined sample, Model III also provides a poor match. The reduction in baryon mass due to the outflows is not matched by reduced SF in the delayed SFH. We also find Model VI provides a poor match to the combined sample, and surprisingly also to HiGH-low. As can be seen in Fig. 3, HIGHlow includes some very actively SF galaxies with small error bars. These sources are more in line with the bursty sources in DGS and 
Table 4. The goodness of fit of each model as expressed by $\left\langle\chi_{\text {eff }}^{2}\right\rangle$ for the combined HRS+HAPLESS+HIGH sample and for HiGH-low. Results for Figs 2,3 and 5 are shown for both samples. $M_{\mathrm{g}} / M_{*}$ was used instead of $f_{\mathrm{g}}$ because for $f_{\mathrm{g}}$ the approximation of a Gaussian error probability distribution is not valid.

\begin{tabular}{|c|c|c|c|c|c|c|}
\hline \multirow[t]{2}{*}{ Galaxy sample } & \multicolumn{3}{|c|}{ Combined } & \multicolumn{3}{|c|}{ HIGH-low } \\
\hline & Fig. 2 & Fig. 3 & Fig. 5 & Fig. 2 & Fig. 3 & Fig. 5 \\
\hline$y$-axis & $M_{\mathrm{d}} / M_{\text {bary }}$ & $\mathrm{SFR} / M_{\text {bary }}$ & $M_{\mathrm{d}} / M_{\mathrm{g}}$ & $M_{\mathrm{d}} / M_{\text {bary }}$ & $\mathrm{SFR} / M_{\text {bary }}$ & $M_{\mathrm{d}} / M_{\mathrm{g}}$ \\
\hline$x$-axis & $M_{\mathrm{g}} / M_{*}$ & $M_{\mathrm{g}} / M_{*}$ & $12+\log (\mathrm{O} / \mathrm{H})$ & $M_{\mathrm{g}} / M_{*}$ & $M_{\mathrm{g}} / M_{*}$ & $12+\log (\mathrm{O} / \mathrm{H})$ \\
\hline Model I & 1.90 & 7.55 & 2.73 & 1.28 & 20.45 & 1.65 \\
\hline Model II & 2.06 & 3.56 & 2.80 & 1.47 & 2.72 & 1.68 \\
\hline Model III & 0.84 & 10.45 & 2.79 & 1.36 & 3.01 & 1.68 \\
\hline Model VI & 3.51 & 6.43 & 0.41 & 0.59 & 3.77 & 1.45 \\
\hline
\end{tabular}

are different from the normal SF galaxies we tried to describe by the reduced delayed SFH in Model VI. Therefore, HiGH-low cannot successfully be described by a single model in Fig. 3 .

For $M_{\mathrm{d}} / M_{\mathrm{g}}$ versus $12+\log (\mathrm{O} / \mathrm{H})$, Table 4 shows Model VI provides the best fit to both HIGH-low and the combined sample. This thus provides further corroboration of dust grain growth being necessary to model the build-up of dust as galaxies evolve. For HIGHlow, the scatter is rather large, and none of the models provides a particularly good fit.

\subsection{Discussion}

We have found that, in order to get satisfactory fits to the observed $M_{\mathrm{d}} / M_{\text {bary }}, \mathrm{SFR} / M_{\text {bary }}, 12+\log (\mathrm{O} / \mathrm{H}), M_{\mathrm{d}} / M_{\mathrm{g}}$ and $M_{\mathrm{d}} / M_{\mathrm{Z}}$ for the different nearby galaxy samples in this work, it is necessary to reduce the $\mathrm{SNe}$ dust contribution (by a factor of 6-100) and include moderate inflows and outflows, dust destruction and moderate grain growth in our models.

The reverse shock in the remnants of SN likely reduces the produced dust (Bianchi \& Schneider 2007; Gall et al. 2011; De Looze et al. 2016). The Todini \& Ferrara (2001) SN dust prescription used in our models does not include a correction for dust destruction by reverse shocks. The need to reduce the $\mathrm{SN}$ dust contribution in Models IV-VI could thus, at least in part, be due to dust destruction by the reverse shock.

There is a growing number of studies that suggest significant amounts of dust grain growth are required to model observations in both high- and low-redshift studies (Dwek, Galliano \& Jones 2007; Matsuura et al. 2009; Michałowski, Watson \& Hjorth 2010; Mattsson \& Andersen 2012; Asano et al. 2013; Grootes et al. 2013; Calura et al. 2014; Rowlands et al. 2014a; Zhukovska 2014; Nozawa et al. 2015; De Cia et al. 2016). On the other hand, Ferrara, Viti \& Ceccarelli (2016) point out the difficulties in obtaining high enough grain growth efficiencies to explain the observations. The subject of dust grain growth thus remains a debated subject. For Models IV-VI, we find grain growth time-scales ranging from $1 \mathrm{Gyr}$ to $200 \mathrm{Myr}$, similar to those quoted for the MW and local galaxies (e.g. Asano et al. 2013; Mattsson \& Andersen 2012). There is also evidence for shorter time-scales (Zhukovska et al. 2008; Draine 2009; Feldmann 2015), which might be more appropriate for the more dust-rich sources at low metallicity or higher metallicity sources. Variations in the dust growth time-scales might also help to explain the differences between dust-rich and dust-poor sources at the same (high) gas fraction. If the reverse shock destroys the majority of the dust grains in SN for all galaxies (and not only the ones modelled well by Models IV-VI), then the higher dust mass sources (which are now fitted by Models I-III) could be explained by shorter dust grain growth time-scales, and high dust mass can be reached in spite of a reduced SN contribution. In this scenario, all galaxies have a strongly reduced $\mathrm{SN}$ dust contribution compared to Todini \& Ferrara (2001), and galaxies with short dust grain growth timescales result in a higher dust content (on the level of Model I), and galaxies with long dust grain growth time-scales (such as in Model VI) will have a lower dust content at high gas fractions.

\subsection{Caveats}

In the previous sections, we have used a range of models to explain the dust properties in the dust-poor low- $Z$ sources, as well as dustrich lower gas-fraction sources. In this section, we discuss potential caveats of our approach.

(i) Dust emissivity. If the dust emissivity is different across the samples, this could explain the reduced $M_{\mathrm{d}} / M_{\mathrm{HI}}$ seen in Fig. 5 and in $M_{\mathrm{d}} / M_{\mathrm{Z}}$ (Fig. 7). For the dust-poor $\mathrm{HIGH}$-low sample to have a dust/metals ratio similar to the HRS and HIGH-high samples (i.e. $\sim 0.4-0.5$ ), $\kappa$ would have to be $\sim 4$ times lower.

(ii) Missing molecular gas. We lack sufficient molecular gas information for the HAPLESS and HIGH samples. To affect our results, the molecular mass would have to be larger than the $\mathrm{HI}$ mass. This does not agree with observed molecular gas masses for the HRS and DGS, nor with the galaxy gas-scaling laws from Saintonge et al. (2011) and Bothwell et al. (2014) for a wide range of stellar masses. These results suggest that $M_{\mathrm{H}_{2}} / M_{\text {bary }}$ is small at all evolutionary phases (see DV17 for more discussion). Using COderived $\mathrm{H}_{2}$ masses for HRS from Boselli et al. (2014), we find that including the molecular gas component does not change the conclusions of our work. At low gas fractions, $M_{\mathrm{H}_{2}} / M_{\mathrm{HI}}$ is large for some sources, and subsequently these will shift to higher gas fractions and higher total gas masses when molecular gas is included. This shift only results in a better fit to the models at low $f_{\mathrm{g}}$ (e.g. Fig. 5).

To study the effects of molecular gas at high gas fraction, we took $M_{\mathrm{H}_{2}}$ for DGS from Rémy-Ruyer et al. (2014). These were derived by converting $\mathrm{CO}$ fluxes using a constant conversion factor $X_{\mathrm{CO}}$, $\mathrm{W}$ (Ackermann et al. 2011) or a metallicity-dependent conversion from CO $X_{\mathrm{CO}, \mathrm{Z}}$ (Schruba et al. 2012). Using $X_{\mathrm{CO}, \mathrm{Mw}}$, we again find $M_{\mathrm{H}_{2}} / M_{\mathrm{HI}}$ is small for all but the lowest gas fractions. The small shift at low gas fractions again results in a better fit with the models. However, if we use $M_{\mathrm{H}_{2}}$ derived using $X_{\mathrm{CO}, \mathrm{Z}}$, we find significantly higher $M_{\mathrm{H}_{2}} / M_{\mathrm{HI}}$ at high gas fractions and thus again a shift towards higher gas masses and gas fractions compared to not including molecular gas masses. For the high gas-fraction sources, this results in a poorer fit to the models assumed here, though the offset does not change our conclusions. Different $X_{\mathrm{CO}, \mathrm{Z}}$ factors (see Bolatto, Wolfire \& Leroy 2013 for a review) lead typically to smaller $M_{\mathrm{H}_{2}}$ 
than for $X_{\mathrm{CO}, \mathrm{Z}}$ from Schruba et al. (2012), and would thus result in smaller offsets.

(iii) Increased dust destruction. We have investigated whether it is possible to explain the observed dust-to-gas properties of the HiGH galaxies by increasing the amount of dust destruction as opposed to reducing the dust production from SNe. We can model increased dust destruction in two ways. First, we can increase the amount of dust which is destroyed per $\mathrm{SNe}$ (by adjusting the value of $m_{\mathrm{ISM}}$ in equation B1) and secondly by adjusting the value of $f_{\mathrm{c}}$ (the fraction of the ISM in the cold phase). With a larger fraction of the ISM in the warm phase, the efficiency of the dust destruction in the galaxy will be increased. We find that changing dust destruction alone cannot match the observed $M_{\mathrm{d}} / M_{\text {bary }}$ and $M_{\mathrm{d}} / M_{\mathrm{g}}$ ratios, since an increased dust destruction does not reduce the dust produced at the high gas fractions $\left(f_{\mathrm{g}}>0.8\right)$. Even an extreme model with $m_{\text {ISM }}=2500 \mathrm{M}_{\odot}$ and $f_{\mathrm{c}}=0.01$ would still require significant $\mathrm{SNe}$ dust reduction to explain the observed dust-to-gas values. Alternatively, if we change $f_{\mathrm{c}}$ to vary with $f_{\mathrm{g}}$, this makes at most a factor of 2 difference to dust destruction, whereas we need a reduction of the dust mass by a factor of $10-100$ at high $f_{\mathrm{g}}$. Therefore, the conclusion of needing a reduced dust yield from SNe first put forward by Zhukovska (2014) is robust to changes in the values of $f_{\mathrm{c}}$ and $m_{\text {ISM }}$. We note that changes in $f_{\mathrm{c}}$ and $m_{\text {ISM }}$ could reduce the offset in $M_{\mathrm{d}} / M_{\text {bary }}$ between the observations and some of the models at low gas fractions.

Given our assumption that gas and dust are uniformly mixed, the dust destruction in our model is proportional to the global dustto-gas ratio. However, in reality $M_{\mathrm{d}} / M_{\mathrm{g}}$ could be higher in SF regions than the global average $M_{\mathrm{d}} / M_{\mathrm{g}}$. More of the SN dust will thus be destroyed before being mixed into the diffuse ISM than is currently the case in our model. Hopkins \& Lee (2016) show that on molecular cloud scales, gas-grain decoupling can lead to fluctuations in the local dust-to-gas ratios. For the highest dust-togas ratios they predict, dust destruction could remove a significant fraction of the dust (even at high gas fractions) compared to the current model. Conversely, dust grain growth would become more efficient. This could thus provide an alternative interpretation for the scatter in $M_{\mathrm{d}} / M_{\text {bary }}$ at high gas fractions and the need to reduce the SN dust contributions. A full treatment of this issue requires spatially resolved chemical evolution modelling, which is outside the scope of this work.

(iv) Initial mass functions. We have also tested how different IMFs change our results. Changing the model IMF to a more bottom-heavy IMF (e.g. Salpeter 1955 or Cappellari et al. 2012), reduces the dust and metals produced in the first generation of stars, which results in a better match of these models compared to the observations (i.e. smaller $M_{\mathrm{d}} / M_{\text {bary }}$ at high gas fractions and smaller $Z$ at low gas fractions). Similarly at high gas fractions, a top-heavy IMF in the model could increase $Z$. But to change the model IMF, we must also scale the observational parameters which have been determined using the Chabrier function. For example, using a topheavy IMF with slope $\alpha=-1.5$ (Cappellari et al. 2012; Madau \& Dickinson 2014), we would have to scale the stellar mass and SFRs by a factor of 0.32 (Michałowski 2015). This results in models that are nearly indistinguishable (in terms of a 'good fit') compared to the scatter in the relations.

\section{CONCLUSIONS}

In this paper, we have brought together the $\mathrm{H}$ I-selected $\mathrm{HIGH}$, dustselected HAPLESS, stellar mass-selected HRS and the metallicityselected DGS sources. Compared to the 126 sources from Rémy-
Ruyer et al. (2014), we have increased the sample size to 382 sources (including 48 DGS sources in both samples). Beyond the 37 DGS sources with $Z<1 / 5 \mathrm{Z}_{\odot}$, we have added a further 67 sources with a metallicity smaller than $1 / 3 \mathrm{Z}_{\odot}$, including 15 sources below $1 / 5 \mathrm{Z}_{\odot}$. Following Zhukovska (2014) and Feldmann (2015), we have investigated the dust trends of these samples using a chemical evolution model (an updated version of Rowlands et al. 2014b and Morgan \& Edmunds 2003). We use the PG16S metallicity calibration, which was found to be the most reliable calibration for the low-metallicity sources, and gas fraction (a proxy for the evolutionary state) to track and constrain the build-up of dust and metals as gas is converted into stars, from very high $\left(f_{\mathrm{g}}=0.97\right)$ to very low $\left(f_{\mathrm{g}}=0.05\right)$ gas fractions. We find that:

(i) DGS sources are selected to have low metallicities, which leads to a selection of very actively SF galaxies. For a given gas fraction or stellar mass, we have found our low $M_{*} \mathrm{HIGH}$ and HAPLESS samples to be more normal in terms of SF properties and metallicity. These samples thus complement the DGS, and provide additional, new information on more normal SF galaxies in the nearby Universe.

(ii) Delayed SFH models are necessary to match the evolution of SFR $/ M_{\text {bary }}$ for our normal SF galaxies.

(iii) We find that low-moderate metallicity galaxies (a) can have dust properties that are consistent with dust production at early stages being dominated by $\mathrm{SNe}$ dust (as in $\mathrm{C} 15$ ), and thus with a linear $M_{\mathrm{d}} / M_{\mathrm{g}}-Z$ relationship and constant $M_{\mathrm{d}} / M_{\mathrm{Z}}$; or (b) have dust masses well below these trends, with a much smaller contribution from $\mathrm{SNe}$ dust. The lowest metallicity sources fall in the latter category and to model them we require a maximum of $0.01-0.16 \mathrm{M}_{\odot}$ of dust per core-collapse SN.

(iv) The dust properties and the observed correlation of $M_{\mathrm{d}} / M_{\mathrm{Z}}-$ $Z$ for low-metallicity sources are well matched when including: (I) reduced stardust contribution by $6-100$, particularly from corecollapse $\mathrm{SNe}$ as the reduced dust component has to act at very high gas fractions. (II) Moderate $(2.5 \times$ SFR) enriched outflows and metal-poor inflows to keep the model metallicity from rising to higher than observed metallicties at low gas fractions. (III) Dust destruction and moderate grain growth (time-scales ranging from 1 Gyr to $200 \mathrm{Myr}$, similar to those quoted for the MW and local galaxies, Draine 2009; Asano et al. 2013; Mattsson \& Andersen 2012; Mattsson et al. 2014). The need for this moderate grain growth is corroborated by the good statistical match of Model VI to the $M_{\mathrm{d}} / M_{\mathrm{g}}-\mathrm{Z}$ relation for both $\mathrm{HIGH}$-low and the combined HRS+HAPLESS+HIGH sample.

(v) As we show that neither the dust-to-metals nor the dust-to-gas ratio are constant during the evolution of a galaxy, we urge caution when using dust as a tracer of gas mass in galaxies (e.g. Eales et al. 2010; Scoville et al. 2014). Assuming a universal value for either is unwise and unlikely to produce reliable results, particularly for low stellar mass systems.

(vi) In our best models, we find that grain growth produces, by mass, 70-93 per cent of the total dust created over the lifetime of these galaxies, and the metallicity at which dust grain growth exceeds stellar dust sources in our model is reached between $7.97<$ $12+\log (\mathrm{O} / \mathrm{H})<8.63$ (or $0.88>f_{\mathrm{g}}>0.53$ ).

We show our Model VI (SN dust contribution reduced by factor 100 , inflows and outflows of $2.5 \times \mathrm{SFR}$, delayed SFH, dust grain growth and destruction) is consistent with all of the observed properties (except the SFR for some rather bursty sources) of the HiGH-low galaxies, the first normal SF population of low stellar mass galaxies studied in this way. Comparing the data and models 
using a Bayesian approach confirms that Model VI provides the best statistical match to the $\mathrm{HIGH}$-low data for the dust-to-baryon ratio against gas fraction and the dust-to-gas ratio against metallicity. For SFR $/ M_{\text {bary }}$, it is not possible to find one model that describes all the $\mathrm{HIGH}$-low data since the intrinsic scatter within the sample is larger than the error bars. When Model VI is combined with a bursty SFH (as shown originally in Zhukovska 2014) and stronger outflows (Feldmann, 2015, Model VII), this scenario is also consistent with the DGS galaxies at $\operatorname{similar} f_{\mathrm{g}}, M_{*}$ and $Z$ without requiring extremely rapid grain growth time-scales and extreme outflows for low-metallicity galaxies.

\section{ACKNOWLEDGEMENTS}

We gratefully acknowledge the anonymous referee for useful suggestions. We thank Suzanne Madden for providing the emission-line tables for the DGS. PDV acknowledges funding from the Fund for Scientific Research Flanders. SPS acknowledges funding from the Science and Technology Facilities Council (STFC) Doctoral Training Grant scheme. HLG acknowledges support from the STFC Consolidated Grant scheme (ST/K000926/1). PDV, HLG, LD, PC and SJM acknowledge support from the European Research Council (ERC) in the form of Consolidator Grant CosmicDust. LD and SJM acknowledge support from the Advanced Investigator programme COSMICISM. GAMA is a joint European-Australasian project based around a spectroscopic campaign using the Anglo-Australian Telescope. The GAMA input catalogue is based on data taken from the SDSS and the UKIRT Infrared Deep Sky Survey. Complementary imaging of the GAMA regions is being obtained by a number of independent survey programmes including GALEX MIS, VST KiDS, VISTA VIKING, WISE, Herschel-ATLAS, GMRT and ASKAP providing UV to radio coverage. GAMA is funded by the STFC (UK), the ARC (Australia), the AAO and the participating institutions. The GAMA website is http://www.gama-survey.org/. This research has made use of GITHUB, ASTROPY 21 (Astropy Collaboration et al. 2013), NUMPY 24 (Walt, Colbert \& Varoquaux 2011), SCIPY 25 and MATPLOTLIB 26 (Hunter 2007).

\section{REFERENCES}

Ackermann M. et al., 2011, ApJ, 726, 81

Asano R. S., Takeuchi T. T., Hirashita H., Inoue A. K., 2013, Earth Planets Space, 65, 213

Asplund M., Grevesse N., Sauval A. J., Scott P., 2009, ARA\&A, 47, 481

Astropy Collaboration et al., 2013, A\&A, 558, A33

Baldwin J. A., Phillips M. M., Terlevich R., 1981, PASP, 93, 5

Barlow M. J. et al., 2010, A\&A, 518, L138

Barnes J. E., Hernquist L., 1992, ARA\&A, 30, 705

Bianchi S., Schneider R., 2007, MNRAS, 378, 973

Blanc G. A., Kewley L., Vogt F. P. A., Dopita M. A., 2015, ApJ, 798, 99

Bolatto A. D., Wolfire M., Leroy A. K., 2013, ARA\&A, 51, 207

Boselli A. et al., 2010, PASP, 122, 261

Boselli A., Hughes T. M., Cortese L., Gavazzi G., Buat V., 2013, A\&A, 550, A114

Boselli A., Cortese L., Boquien M., 2014, A\&A, 564, A65

Bothwell M. S. et al., 2014, MNRAS, 445, 2599

Bourne N. et al., 2012, MNRAS, 421, 3027

Bourne N. et al., 2016, MNRAS, 462, 1714

Bresolin F., Kennicutt R. C., 2015, MNRAS, 454, 3664

Calura F., Gilli R., Vignali C., Pozzi F., Pipino A., Matteucci F., 2014, MNRAS, 438, 2765

Cappellari M. et al., 2012, Nature, 484, 485

Cardelli J. A., Clayton G. C., Mathis J. S., 1989, ApJ, 345, 245

Chabrier G., 2003, PASP, 115, 763

Clark C. J. R. et al., 2015, MNRAS, 452, 397
Clark C. J. R., Schofield S. P., Gomez H. L., Davies J. I., 2016, MNRAS, 459, 1646

Cortese L. et al., 2012, A\&A, 540, A52

Cortese L. et al., 2014, MNRAS, 440, 942

da Cunha E., Charlot S., Elbaz D., 2008, MNRAS, 388, 1595

Davies J. I. et al., 2014, MNRAS, 438, 1922

De Cia A., Ledoux C., Savaglio S., Schady P., Vreeswijk P. M., 2013, A\&A, 560, A88

De Cia A., Ledoux C., Mattsson L., Petitjean P., Srianand R., Gavignaud I., Jenkins E. B., 2016, A\&A, 596, A97

De Looze I. et al., 2016, MNRAS, 459, 3900

De Vis P. et al., 2017, MNRAS, 464, 4680

Draine B. T., 2009, in Henning T., Grün E., Steinacker J., eds, ASP Conf. Ser. Vol. 414, Cosmic Dust - Near and Far. Astron. Soc. Pac., San Francisco, p. 453

Dunne L., Eales S., Ivison R., Morgan H., Edmunds M., 2003, Nature, 424, 285

Dunne L. et al., 2009, MNRAS, 394, 1307

Dunne L. et al., 2011, MNRAS, 417, 1510

Dwek E., Cherchneff I., 2011, ApJ, 727, 63

Dwek E., Galliano F., Jones A. P., 2007, ApJ, 662, 927

Eales S. et al., 2010, PASP, 122, 499

Eskew M., Zaritsky D., Meidt S., 2012, AJ, 143, 139

Feldmann R., 2015, MNRAS, 449, 3274

Ferrara A., Viti S., Ceccarelli C., 2016, MNRAS, 463, L112

Ferrarotti A. S., Gail H.-P., 2006, A\&A, 447, 553

Ferreras I., Silk J., 2000, ApJ, 532, 193

Fisher D. B. et al., 2014, Nature, 505, 186

Gall C., Andersen A. C., Hjorth J., 2011, A\&A, 528, A14

Gall C. et al., 2014, Nature, 511, 326

Garnett D. R., 1992, AJ, 103, 1330

Giovanelli R. et al., 2005, AJ, 130, 2598

Gomez H. L. et al., 2012, ApJ, 760, 96

Gordon Y. A. et al., 2016, MNRAS, 465, 2671

Grootes M. W. et al., 2013, ApJ, 766, 59

Guseva N. G., Izotov Y. I., Thuan T. X., 2000, ApJ, 531, 776

Guseva N. G., Papaderos P., Izotov Y. I., Green R. F., Fricke K. J., Thuan T. X., Noeske K. G., 2003, A\&A, 407, 91

Guseva N. G., Izotov Y. I., Papaderos P., Fricke K. J., 2007, A\&A, 464, 885

Guseva N. G., Izotov Y. I., Fricke K. J., Henkel C., 2012, A\&A, 541, A115

Haynes M. P. et al., 2011, AJ, 142, 170

Herrera-Camus R. et al., 2012, ApJ, 752, 112

Hopkins P. F., Lee H., 2016, MNRAS, 456, 4174

Hopkins A. M. et al., 2013, MNRAS, 430, 2047

Hunter J. D., 2007, Comput. Sci. Eng., 9, 90

Hunter D. A. et al., 2012, AJ, 144, 134

Indebetouw R. et al., 2014, ApJ, 782, L2

Inoue A. K., 2003, PASJ, 55, 901

Izotov Y. I., Thuan T. X., 1998, ApJ, 500, 188

Izotov Y. I., Thuan T. X., 2004, ApJ, 602, 200

Izotov Y. I., Thuan T. X., Lipovetsky V. A., 1994, ApJ, 435, 647

Izotov Y. I., Thuan T. X., Lipovetsky V. A., 1997, ApJS, 108, 1

Izotov Y. I., Stasińska G., Meynet G., Guseva N. G., Thuan T. X., 2006, A\&A, 448, 955

Izotov Y. I., Thuan T. X., Stasińska G., 2007, ApJ, 662, 15

James A., Dunne L., Eales S., Edmunds M. G., 2002, MNRAS, 335, 753

Kauffmann G. et al., 2003, MNRAS, 346, 1055

Kennicutt R. C., Jr, et al., 2009, ApJ, 703, 1672

Kewley L. J., Ellison S. L., 2008, ApJ, 681, 1183

Kobulnicky H. A., Skillman E. D., 1997, ApJ, 489, 636

Kobulnicky H. A., Kennicutt Jr R. C., Pizagno J. L., 1999, ApJ, 514, 544

Kong X., Cheng F. Z., 2002, A\&A, 389, 845

Lara-López M. A. et al., 2010, A\&A, 521, L53

Lee H., Skillman E. D., 2004, ApJ, 614, 698

Lee S.-K., Ferguson H. C., Somerville R. S., Wiklind T., Giavalisco M., 2010, ApJ, 725, 1644

López-Sánchez Á. R., Esteban C., Rodríguez M., 2004, ApJS, 153, 243

Madau P., Dickinson M., 2014, ARA\&A, 52, 415 
Madden S. C. et al., 2013, PASP, 125, 600

Maeder A., 1992, A\&A, 264, 105

Magrini L., Gonçalves D. R., 2009, MNRAS, 398, 280

Mancini M., Schneider R., Graziani L., Valiante R., Dayal P., Maio U., Ciardi B., Hunt L. K., 2015, MNRAS, 451, L70

Mannucci F., Cresci G., Maiolino R., Marconi A., Gnerucci A., 2010, MNRAS, 408, 2115

Marino R. A. et al., 2013, A\&A, 559, A114

Masegosa J., Moles M., Campos-Aguilar A., 1994, ApJ, 420, 576

Matsuura M. et al., 2009, MNRAS, 396, 918

Matsuura M. et al., 2011, Science, 333, 1258

Mattsson L., Andersen A. C., 2012, MNRAS, 423, 38

Mattsson L. et al., 2014, MNRAS, 444, 797

Meyer M. J. et al., 2004, MNRAS, 350, 1195

Michałowski M. J., 2015, A\&A, 577, A80

Michałowski M. J., Watson D., Hjorth J., 2010, ApJ, 712, 942

Morales-Luis A. B., Pérez-Montero E., Sánchez Almeida J., Muñoz-Tuñón C., 2014, ApJ, 797, 81

Morgan H. L., Edmunds M. G., 2003, MNRAS, 343, 427

Moustakas J., Kennicutt Jr. R. C., Tremonti C. A., Dale D. A., Smith J.-D. T., Calzetti D., 2010, ApJS, 190, 233

Nozawa T., Asano R. S., Hirashita H., Takeuchi T. T., 2015, MNRAS, 447, L16

Peimbert A., Peimbert M., Ruiz M. T., 2005, ApJ, 634, 1056

Pettini M., Pagel B. E. J., 2004, MNRAS, 348, L59

Pilbratt G. L. et al., 2010, A\&A, 518, L1

Pilyugin L. S., Grebel E. K., 2016, MNRAS, 457, 3678

Pilyugin L. S., Thuan T. X., 2005, ApJ, 631, 231

Popescu C. C., Hopp U., 2000, A\&AS, 142, 247

Popping G., Somerville R. S., Trager S. C., 2014, MNRAS, 442, 2398

Popping G., Somerville R. S., Galametz M., 2016, MNRAS, preprint (arXiv:1609.08622)

Pustilnik S. A., Kniazev A. Y., Pramskij A. G., Ugryumov A. V., 2003, Ap\&SS, 284, 795

Rémy-Ruyer A. et al., 2013, A\&A, 557, A95

Rémy-Ruyer A. et al., 2014, A\&A, 563, A31

Rémy-Ruyer A. et al., 2015, A\&A, 582, A121

Rho J. et al., 2008, ApJ, 673, 271

Romano D., Karakas A. I., Tosi M., Matteucci F., 2010, A\&A, 522, A32

Rowlands K. et al., 2014a, MNRAS, 441, 1017

Rowlands K., Gomez H. L., Dunne L., Aragón-Salamanca A., Dye S., Maddox S., da Cunha E., Werf P. v. d., 2014b, MNRAS, 441, 1040
Saintonge A. et al., 2011, MNRAS, 415, 32

Salpeter E. E., 1955, ApJ, 121, 161

Sánchez S. F. et al., 2016, Rev. Mex. Astron. Astrofis., 52, 21

Sargent B. A. et al., 2010, ApJ, 716, 878

Sarzi M. et al., 2006, MNRAS, 366, 1151

Schaller G., Schaerer D., Meynet G., Maeder A., 1992, A\&AS, 96, 269

Schruba A. et al., 2012, AJ, 143, 138

Scoville N. et al., 2014, ApJ, 783, 84

Skillman E. D., Kennicutt Jr R. C., 1993, ApJ, 411, 655

Skillman E. D., Côté S., Miller B. W., 2003, AJ, 125, 610

Smith D. J. B. et al., 2012, MNRAS, 427, 703

Stasińska G., 2005, A\&A, 434, 507

Thomas D. et al., 2013, MNRAS, 431, 1383

Tinsley B. M., 1980, Fund. Cosmic Phys., 5, 287

Todini P., Ferrara A., 2001, MNRAS, 325, 726

Tremonti C. A. et al., 2004, ApJ, 613, 898

Valiante E. et al., 2016, MNRAS, 462, 3146

van den Hoek L. B., Groenewegen M. A. T., 1997, A\&AS, 123, 305

van Zee L., Haynes M. P., Salzer J. J., Broeils A. H., 1996, AJ, 112, 129

Ventura P. et al., 2012, MNRAS, 424, 2345

Walt S. v. d., Colbert S. C., Varoquaux G., 2011, Comput. Sci. Eng., 13, 22

Yin J., Hou J. L., Prantzos N., Boissier S., Chang R. X., Shen S. Y., Zhang B., 2009, A\&A, 505, 497

Zafar T., Watson D., 2013, A\&A, 560, A26

Zhukovska S., 2014, A\&A, 562, A76

Zhukovska S., Gail H.-P., Trieloff M., 2008, A\&A, 479, 453

\section{APPENDIX A: EMISSION LINES AND GALAXY PROPERTIES}

The basic galaxy properties for the HIGH and HAPLESS galaxies are listed in Table A1 and their metallicities in Table A2. SFRs, and stellar and dust masses were derived using MAGPHYS (see DV17 for details). Metallicities were derived for four different calibrations, using a weighted average of the metallicities from individual $\mathrm{H}$ II regions within the galaxy (Section 3). The emission lines for each $\mathrm{H}$ II region in the HIGH and HAPLESS galaxies are listed in Table A3. For DGS, emission lines from literature and derived metallicities are provided in Table A4.

Table A1. Basic properties for the $\mathrm{H}$ I-selected HIGH sample (DV17) and dust-selected HAPLESS sample (C15). The check marks indicate which sample the source is part of (24 sources are included in both samples).

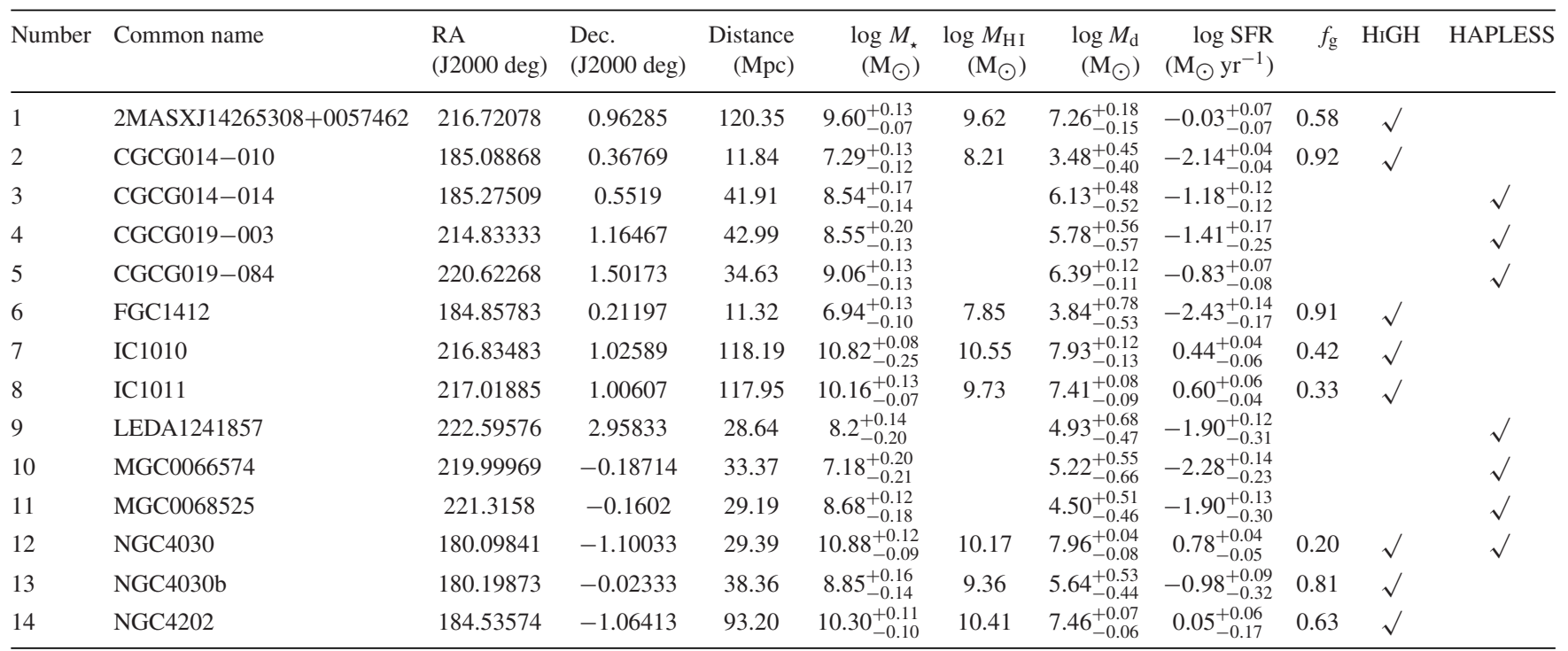


Table A1 - continued

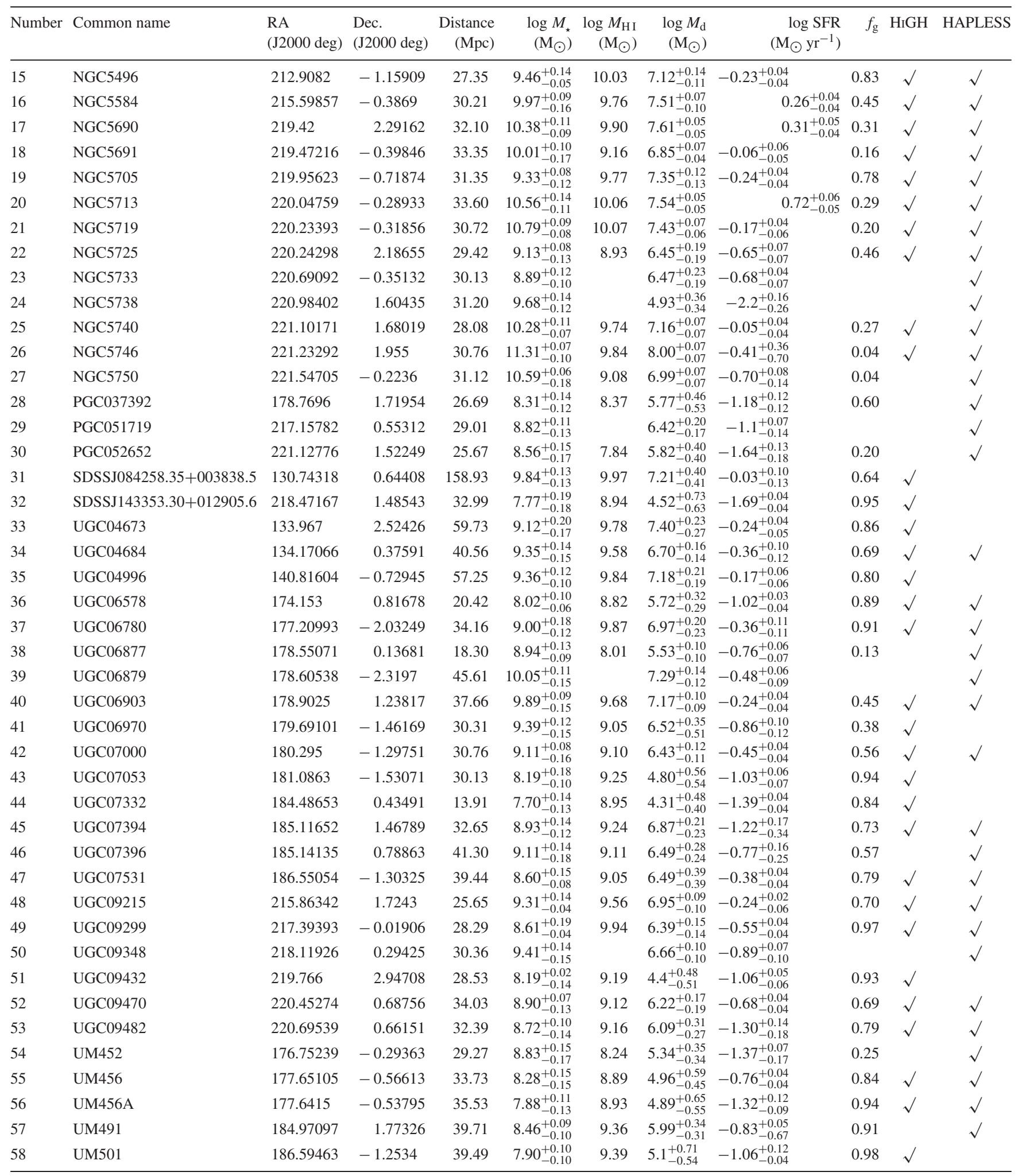


Table A2. Metallicity measurements in the form $12+\log (\mathrm{O} / \mathrm{H})$ for the $\mathrm{H}$ I-selected $\mathrm{HIGH}$ sample (DV17) and dust-selected HAPLESS sample (C15). The check marks indicate which sample the source is part of (24 sources are included in both samples).

\begin{tabular}{|c|c|c|c|c|c|c|c|}
\hline \multirow[t]{2}{*}{ Number } & \multirow[t]{2}{*}{ Common name } & \multicolumn{4}{|c|}{$12+\log (\mathrm{O} / \mathrm{H})$} & \multirow[t]{2}{*}{$\mathrm{HIGH}$} & \multirow[t]{2}{*}{ HAPLESS } \\
\hline & & $\mathrm{O} 3 \mathrm{~N} 2$ & $\mathrm{~N} 2$ & PG16S & KE08/T04 & & \\
\hline 1 & 2MASXJ14265308+0057462 & $8.53_{-0.12}^{+0.12}$ & $8.53_{-0.12}^{+0.12}$ & $8.37_{-0.12}^{+0.12}$ & $8.77_{-0.13}^{+0.13}$ & $\sqrt{ }$ & \\
\hline 3 & CGCG014-014 & $8.25_{-0.14}^{+0.13}$ & $8.15_{-0.13}^{+0.12}$ & $7.75_{-0.26}^{+0.37}$ & $8.42_{-0.14}^{+0.13}$ & & $\sqrt{ }$ \\
\hline 4 & CGCG019-003 & $8.21_{-0.12}^{+0.12}$ & $8.22_{-0.12}^{+0.12}$ & $8.14_{-0.13}^{+0.12}$ & $8.36_{-0.12}^{+0.12}$ & & $\sqrt{ }$ \\
\hline 5 & CGCG019-084 & $8.59_{-0.13}^{+0.13}$ & $8.50_{-0.12}^{+0.12}$ & & $8.84_{-0.13}^{+0.13}$ & & $\sqrt{ }$ \\
\hline 7 & IC1010 & & & & & $\sqrt{ }$ & \\
\hline 8 & IC1011 & $8.73_{-0.12}^{+0.12}$ & $8.77_{-0.20}^{+0.16}$ & $8.60_{-0.12}^{+0.12}$ & $9.01_{-0.13}^{+0.13}$ & $\sqrt{ }$ & \\
\hline 9 & LEDA1241857 & $8.34_{-0.12}^{+0.12}$ & $8.29_{-0.12}^{+0.12}$ & $8.19_{-0.12}^{+0.12}$ & $8.54_{-0.12}^{+0.12}$ & & $\sqrt{ }$ \\
\hline 10 & MGC0066574 & & & & & & $\sqrt{ }$ \\
\hline 11 & MGC0068525 & & & & & & $\sqrt{ }$ \\
\hline 15 & NGC5496 & $8.34_{-0.10}^{+0.10}$ & $8.29_{-0.10}^{+0.10}$ & $8.19_{-0.10}^{+0.10}$ & $8.54_{-0.10}^{+0.10}$ & $\sqrt{ }$ & $\sqrt{ }$ \\
\hline 16 & NGC5584 & $8.49_{-0.10}^{+0.10}$ & $8.43_{-0.10}^{+0.10}$ & $8.36_{-0.10}^{+0.10}$ & $8.72_{-0.10}^{+0.10}$ & $\sqrt{ }$ & $\sqrt{ }$ \\
\hline 17 & NGC5690 & $8.76_{-0.11}^{+0.11}$ & $8.58_{-0.11}^{+0.11}$ & $8.48_{-0.11}^{+0.11}$ & $9.04_{-0.11}^{+0.11}$ & $\sqrt{ }$ & $\sqrt{ }$ \\
\hline 18 & NGC5691 & $8.57_{-0.10}^{+0.10}$ & $8.54_{-0.10}^{+0.10}$ & $8.42_{-0.10}^{+0.10}$ & $8.82_{-0.10}^{+0.10}$ & $\sqrt{ }$ & $\sqrt{ }$ \\
\hline 19 & NGC5705 & $8.56_{-0.11}^{+0.11}$ & $8.48_{-0.11}^{+0.11}$ & $8.30_{-0.11}^{+0.11}$ & $8.81_{-0.12}^{+0.12}$ & $\sqrt{ }$ & $\sqrt{ }$ \\
\hline 20 & NGC5713 & $8.76_{-0.10}^{+0.10}$ & $8.63_{-0.10}^{+0.10}$ & $8.53_{-0.10}^{+0.10}$ & $9.05_{-0.10}^{+0.10}$ & $\sqrt{ }$ & $\sqrt{ }$ \\
\hline 21 & NGC5719 & & & & & $\sqrt{ }$ & $\sqrt{ }$ \\
\hline 22 & NGC5725 & $8.42_{-0.10}^{+0.10}$ & $8.41_{-0.10}^{+0.10}$ & $8.31_{-0.10}^{+0.10}$ & $8.65_{-0.10}^{+0.10}$ & $\sqrt{ }$ & $\sqrt{ }$ \\
\hline 23 & NGC5733 & $8.28_{-0.11}^{+0.10}$ & $8.28_{-0.11}^{+0.11}$ & $8.18_{-0.11}^{+0.11}$ & $8.48_{-0.11}^{+0.11}$ & & $\sqrt{ }$ \\
\hline 24 & NGC5738 & & & & & & $\sqrt{ }$ \\
\hline 32 & SDSSJ143353.30+012905.6 & & & & & $\sqrt{ }$ & \\
\hline 33 & UGC04673 & $8.50_{-0.11}^{+0.11}$ & $8.45_{-0.11}^{+0.11}$ & $8.36_{-0.11}^{+0.11}$ & $8.75_{-0.12}^{+0.12}$ & $\sqrt{ }$ & \\
\hline 34 & UGC04684 & $8.58_{-0.12}^{+0.11}$ & $8.48_{-0.12}^{+0.11}$ & & $8.84_{-0.13}^{+0.13}$ & $\sqrt{ }$ & $\sqrt{ }$ \\
\hline 35 & UGC04996 & $8.45_{-0.11}^{+0.11}$ & $8.36_{-0.11}^{+0.11}$ & $8.18_{-0.11}^{+0.11}$ & $8.68_{-0.12}^{+0.12}$ & $\sqrt{ }$ & \\
\hline 36 & UGC06578 & $8.04_{-0.11}^{+0.11}$ & $7.99_{-0.11}^{+0.11}$ & $7.87_{-0.12}^{+0.12}$ & $8.25_{-0.13}^{+0.13}$ & $\sqrt{ }$ & $\sqrt{ }$ \\
\hline 37 & UGC06780 & $8.42_{-0.11}^{+0.11}$ & $8.32_{-0.11}^{+0.11}$ & $8.06_{-0.11}^{+0.11}$ & $8.64_{-0.12}^{+0.12}$ & $\sqrt{ }$ & $\sqrt{ }$ \\
\hline 38 & UGC06877 & $8.58_{-0.12}^{+0.12}$ & $8.53_{-0.12}^{+0.12}$ & $8.52_{-0.12}^{+0.12}$ & $8.83_{-0.12}^{+0.12}$ & & $\sqrt{ }$ \\
\hline 39 & UGC06879 & & & & & & $\sqrt{ }$ \\
\hline 40 & UGC06903 & $8.59_{-0.11}^{+0.12}$ & $8.49_{-0.12}^{+0.12}$ & $8.33_{-0.12}^{+0.12}$ & $8.85_{-0.12}^{+0.12}$ & $\sqrt{ }$ & $\sqrt{ }$ \\
\hline 41 & UGC06970 & $8.54_{-0.13}^{+0.13}$ & $8.45_{-0.16}^{+0.16}$ & $8.16_{-0.19}^{+0.16}$ & $8.79_{-0.15}^{+0.15}$ & $\sqrt{ }$ & \\
\hline 42 & UGC07000 & $8.39_{-0.10}^{+0.10}$ & $8.34_{-0.10}^{+0.10}$ & $8.25_{-0.10}^{+0.10}$ & $8.59_{-0.11}^{+0.11}$ & $\sqrt{ }$ & $\sqrt{ }$ \\
\hline 43 & UGC07053 & $8.21_{-0.11}^{+0.11}$ & $8.15_{-0.11}^{+0.11}$ & $7.88_{-0.12}^{+0.12}$ & $8.36_{-0.12}^{+0.12}$ & $\sqrt{ }$ & \\
\hline 44 & UGC07332 & & & & & $\sqrt{ }$ & \\
\hline 45 & UGC07394 & $8.28_{-0.11}^{+0.11}$ & $8.20_{-0.11}^{+0.11}$ & $7.94_{-0.13}^{+0.12}$ & $8.47_{-0.12}^{+0.12}$ & $\sqrt{ }$ & $\sqrt{ }$ \\
\hline 46 & UGC07396 & $8.44_{-0.12}^{+0.12}$ & $8.39_{-0.12}^{+0.12}$ & $8.26_{-0.12}^{+0.12}$ & $8.68_{-0.12}^{+0.12}$ & & $\sqrt{ }$ \\
\hline 47 & UGC07531 & & & & & $\sqrt{ }$ & $\sqrt{ }$ \\
\hline 48 & UGC09215 & $8.40_{-0.10}^{+0.10}$ & $8.37_{-0.10}^{+0.10}$ & $8.29_{-0.10}^{+0.10}$ & $8.62_{-0.11}^{+0.11}$ & $\sqrt{ }$ & $\sqrt{ }$ \\
\hline
\end{tabular}


Table A2 - continued

\begin{tabular}{|c|c|c|c|c|c|c|c|}
\hline Number & Common name & \multicolumn{4}{|c|}{$12+\log (\mathrm{O} / \mathrm{H})$} & $\mathrm{HIGH}$ & HAPLESS \\
\hline 49 & UGC09299 & $8.39_{-0.11}^{+0.11}$ & $8.36_{-0.11}^{+0.11}$ & $8.25_{-0.11}^{+0.11}$ & $8.60_{-0.12}^{+0.12}$ & $\sqrt{ }$ & $\sqrt{ }$ \\
\hline 51 & UGC09432 & $8.25_{-0.12}^{+0.12}$ & $8.21_{-0.12}^{+0.12}$ & $8.06_{-0.16}^{+0.12}$ & $8.42_{-0.13}^{+0.13}$ & $\sqrt{ }$ & \\
\hline 52 & UGC09470 & $8.13_{-0.12}^{+0.12}$ & $8.15_{-0.12}^{+0.12}$ & $7.94_{-0.12}^{+0.12}$ & $8.22_{-0.13}^{+0.13}$ & $\sqrt{ }$ & $\sqrt{ }$ \\
\hline 55 & UM456 & $8.04_{-0.11}^{+0.11}$ & $8.05_{-0.11}^{+0.11}$ & $7.81_{-0.11}^{+0.11}$ & $8.20_{-0.13}^{+0.13}$ & $\sqrt{ }$ & $\sqrt{ }$ \\
\hline 56 & UM456A & $8.06_{-0.11}^{+0.11}$ & $8.04_{-0.11}^{+0.11}$ & $7.72_{-0.11}^{+0.11}$ & $8.10_{-0.13}^{+0.13}$ & $\sqrt{ }$ & $\sqrt{ }$ \\
\hline 57 & UM491 & $8.25_{-0.12}^{+0.12}$ & $8.24_{-0.12}^{+0.12}$ & $8.18_{-0.12}^{+0.12}$ & $8.42_{-0.12}^{+0.12}$ & & $\sqrt{ }$ \\
\hline 58 & UM501 & & & & & $\sqrt{ }$ & \\
\hline
\end{tabular}

\section{APPENDIX B: DUST MASS EQUATION}

Here, we reproduce the equation for the dust mass evolution, $M_{\mathrm{d}}$, for the chemical evolution model used in this work:

$$
\begin{aligned}
\frac{\mathrm{d}\left(M_{\mathrm{d}}\right)}{\mathrm{d} t}= & \int_{m_{\tau_{\mathrm{m}}}}^{m_{\mathrm{U}}}\left(\left[m-m_{\mathrm{R}}(m)\right] Z\left(t-\tau_{\mathrm{m}}\right) \delta_{\text {lims }}+m p_{z} \delta_{\mathrm{dust}}\right) \\
& \times \psi\left(t-\tau_{\mathrm{m}}\right) \phi(m) \mathrm{d} m-\left(M_{\mathrm{d}} / M_{\mathrm{g}}\right) \psi(t) \\
& -\left(1-f_{\mathrm{c}}\right) \frac{M_{\mathrm{d}}}{\tau_{\mathrm{dest}}}+f_{\mathrm{c}}\left(1-\frac{M_{\mathrm{d}}}{M_{\mathrm{g}}}\right) \frac{M_{\mathrm{d}}}{\tau_{\text {grow }}} \\
& +M_{\mathrm{d}, \mathrm{i}}+\left(\frac{M_{\mathrm{d}}}{M_{\mathrm{g}}}\right)_{\mathrm{I}} I(t)-\left(\frac{M_{\mathrm{d}}}{M_{\mathrm{g}}}\right)_{\mathrm{O}} O(t) .
\end{aligned}
$$

$M_{\mathrm{g}}$ is the gas mass, $\psi(t)$ is the SFR, $\phi(m)$ is the stellar IMF, $Z$ is the metal mass fraction defined as $M_{\mathrm{Z}} / M_{\mathrm{g}}$ and $m_{\mathrm{R}}$ is the remnant mass of a star of mass $m$ (Ferreras \& Silk 2000). The first term accounts for dust formed in stars and SNe. This includes metals re-released by stars after they die, and newly synthesized metals ejected in winds and $\mathrm{SNe}$. The second term describes the removal of dust due to astration and the grain destruction and growth timescales are given in terms three and four. The fifth term allows us to include primordial dust in the galaxy, for example associated with Pop III stars, we set this to zero (Rowlands et al. 2014b). Finally, $I(t)$ and $O(t)$ are simple parametrizations of dust removed or contributed via inflows and outflows. The lifetime $\tau_{\mathrm{m}}$ of stars with initial mass $m$ is derived using the model in Schaller et al.
(1992) and yields for LIMS and massive stars are taken from van den Hoek \& Groenewegen (1997) and Maeder (1992), respectively. A full discussion on the effect of using different yields can be seen in Rowlands et al. (2014b) and Romano et al. (2010).

\section{APPENDIX C: COMPARISON OF MODELS WITH DWARF GALAXY SURVEY}

We can model the properties of the DGS sources by including strong inflows and outflows (Feldmann 2015) and a bursty SFH (Zhukovska 2014) in the chemical evolution (Model VII). The results are shown in Fig. C1 using the original DGS metallicities (transparent triangles), and the revised PG16S metallicities derived in this work. In the top-left panel, we compare the $M_{\mathrm{d}} / M_{\text {bary }}$ of the DGS with Model VII (as we did with the HRS, HiGH and HAPLESS in Fig. 2). Model VII matches the observed trend well. In the top-right panel of Fig. C1, we compare the predicted SFR/ $M_{\text {bary }}$ with gas fraction for Model VII. Here, we see that the bursty model is required to explain the elevated SFR/ $M_{\text {bary }}$ of the DGS galaxies compared to the HAPLESS, HRS and HIGH samples. In the bottom-left panel, we find the observed metallicities for DGS tend to be lower than for the other samples and are well matched by Model VII, due to including strong inflows and outflows at a rate of four times the SFR. 


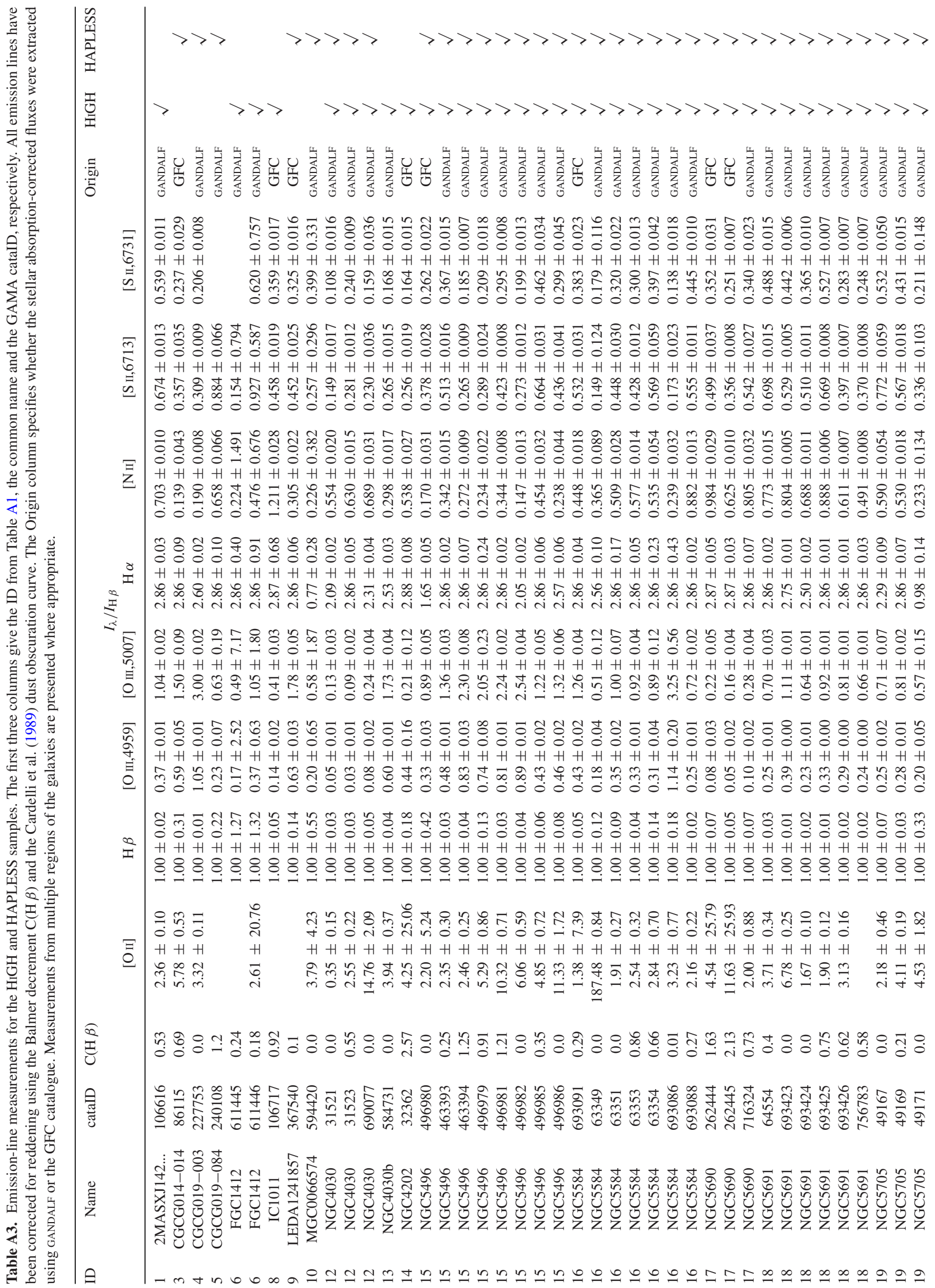




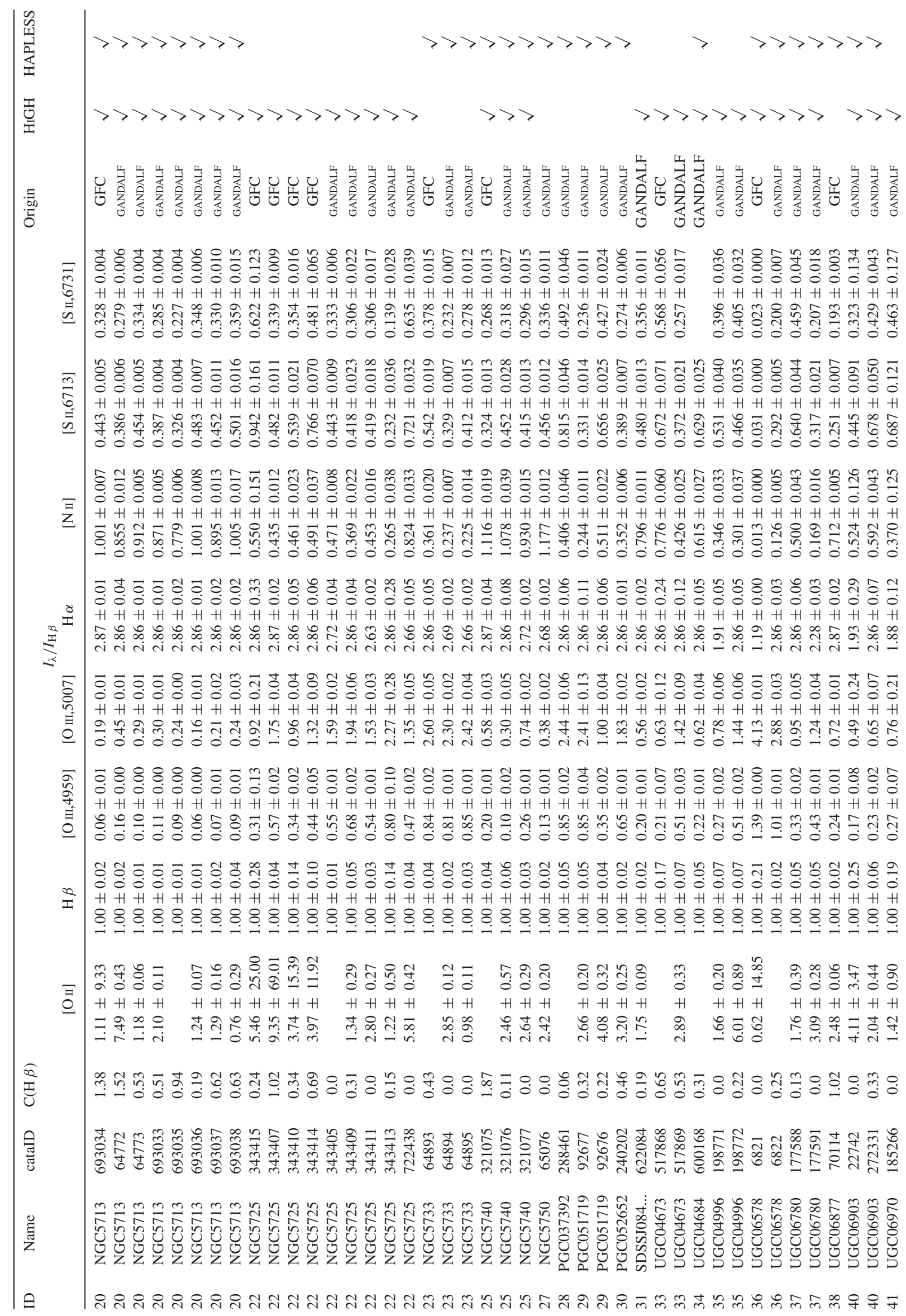




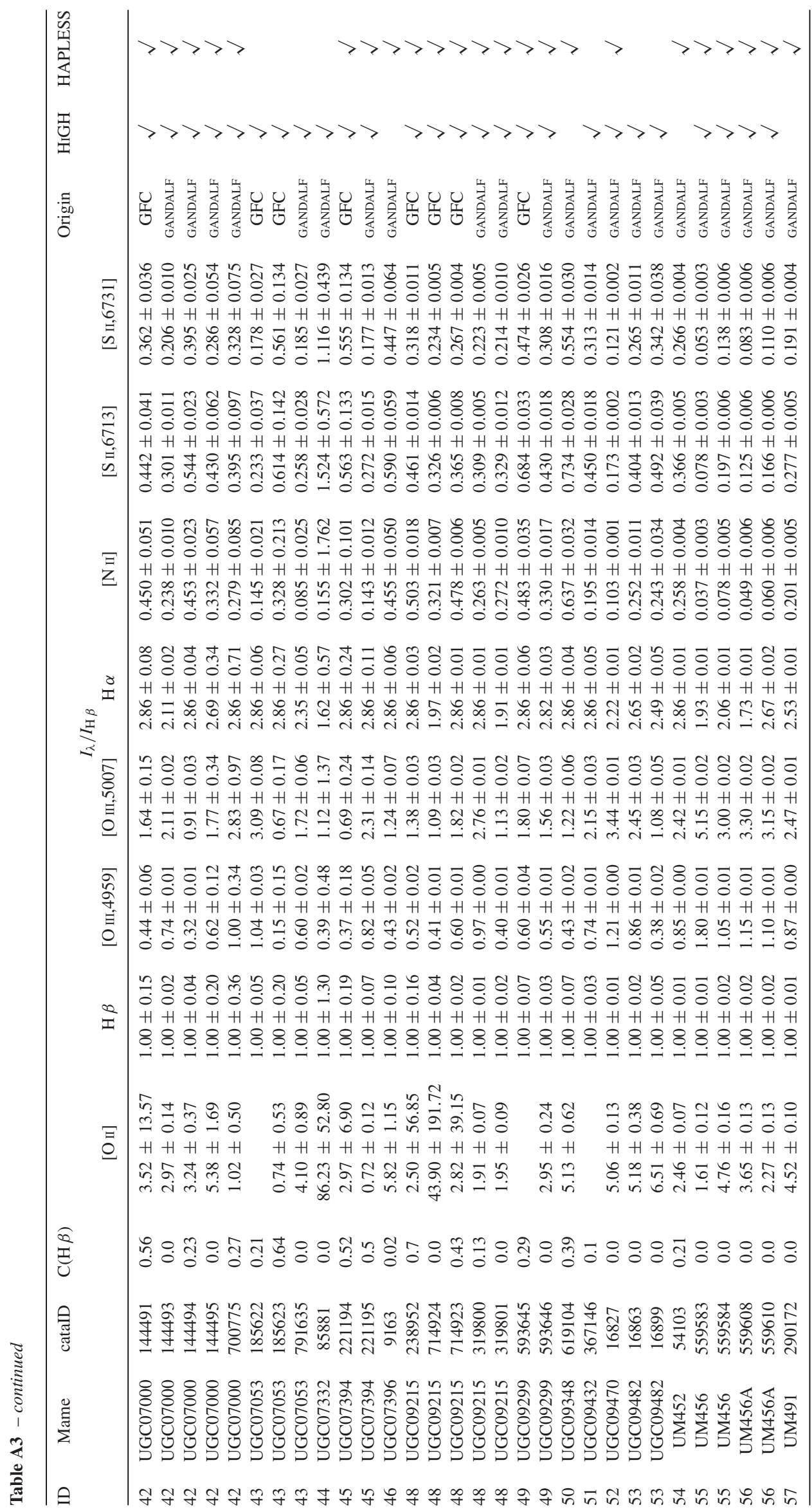




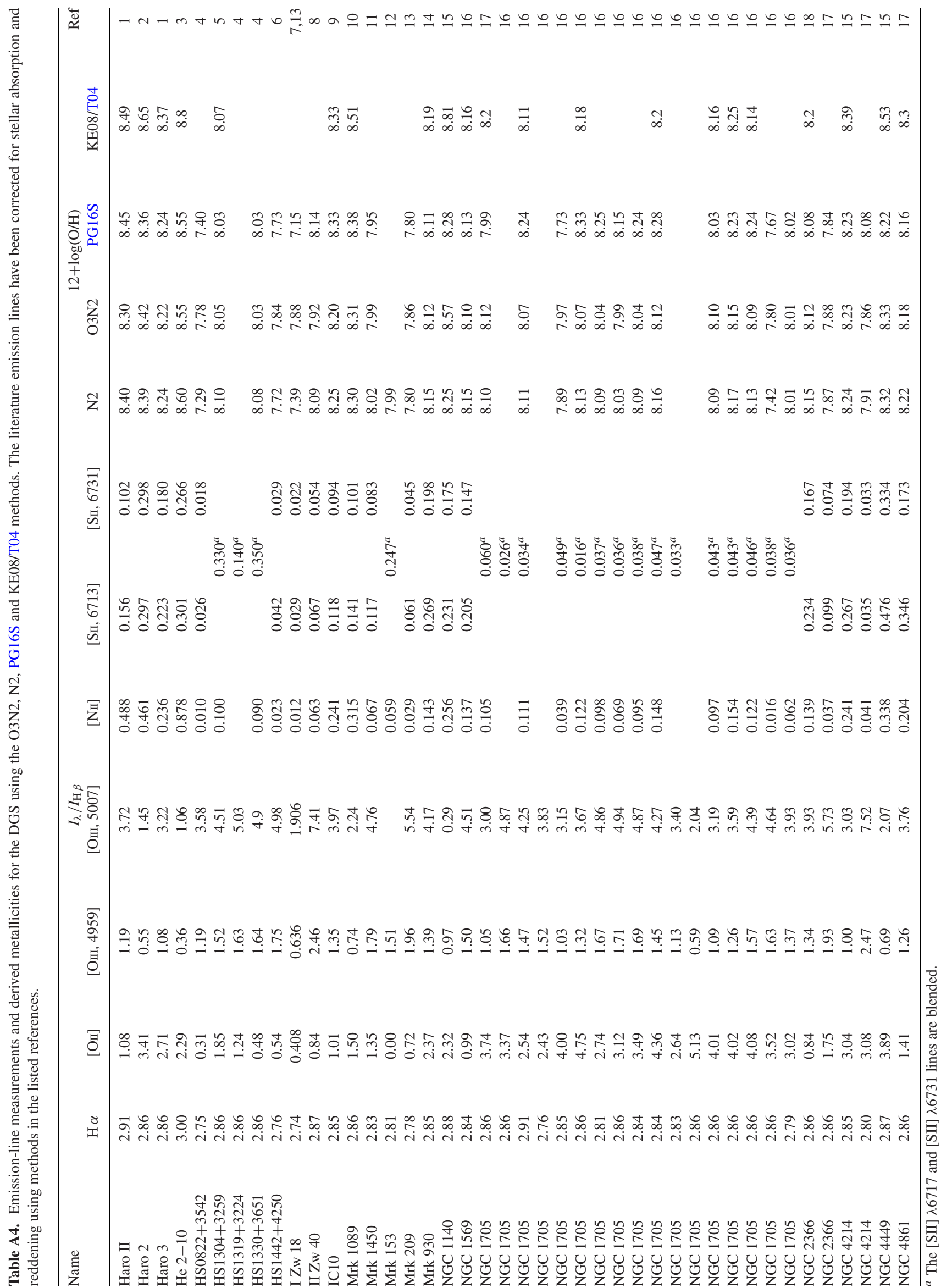




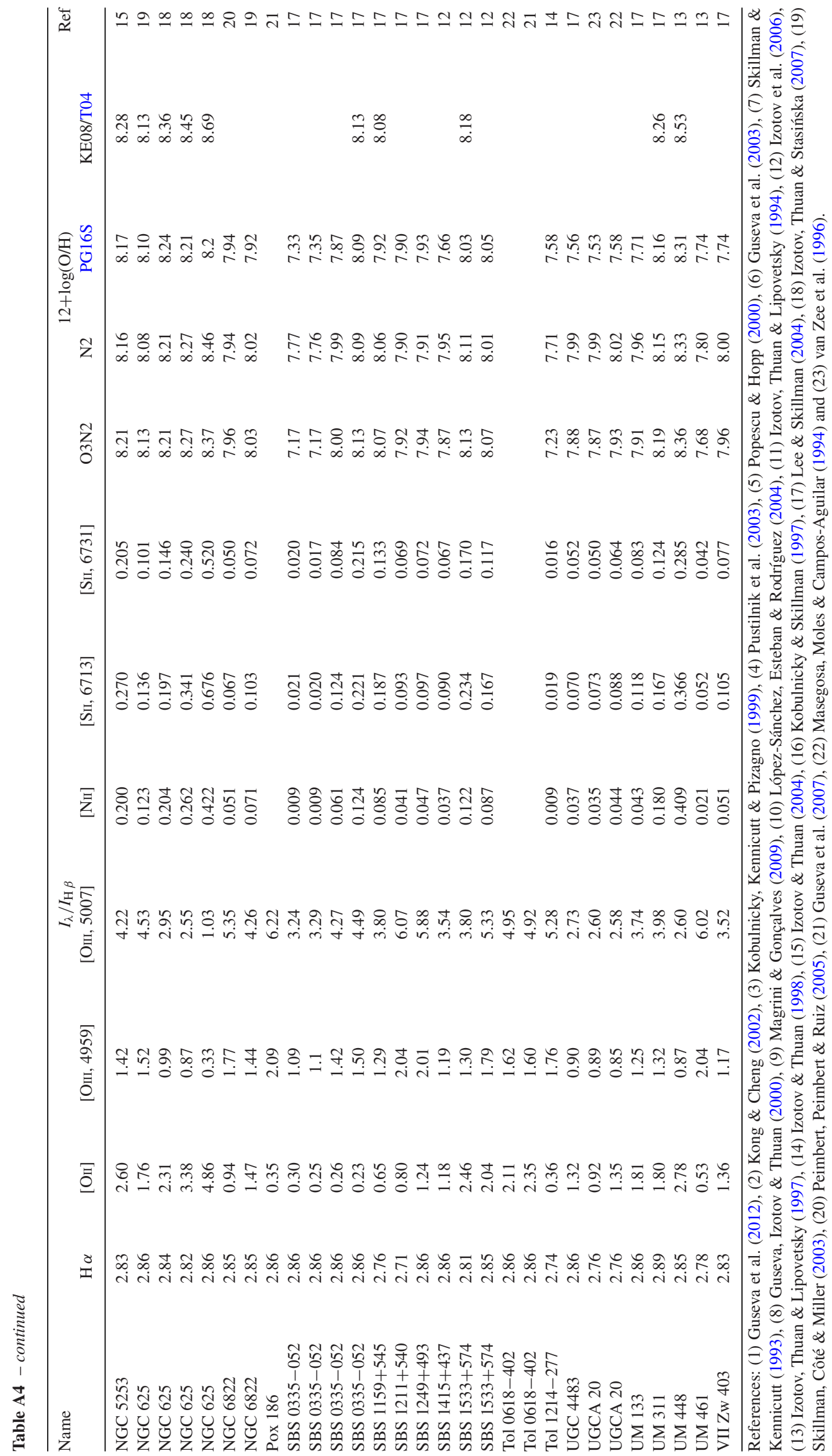



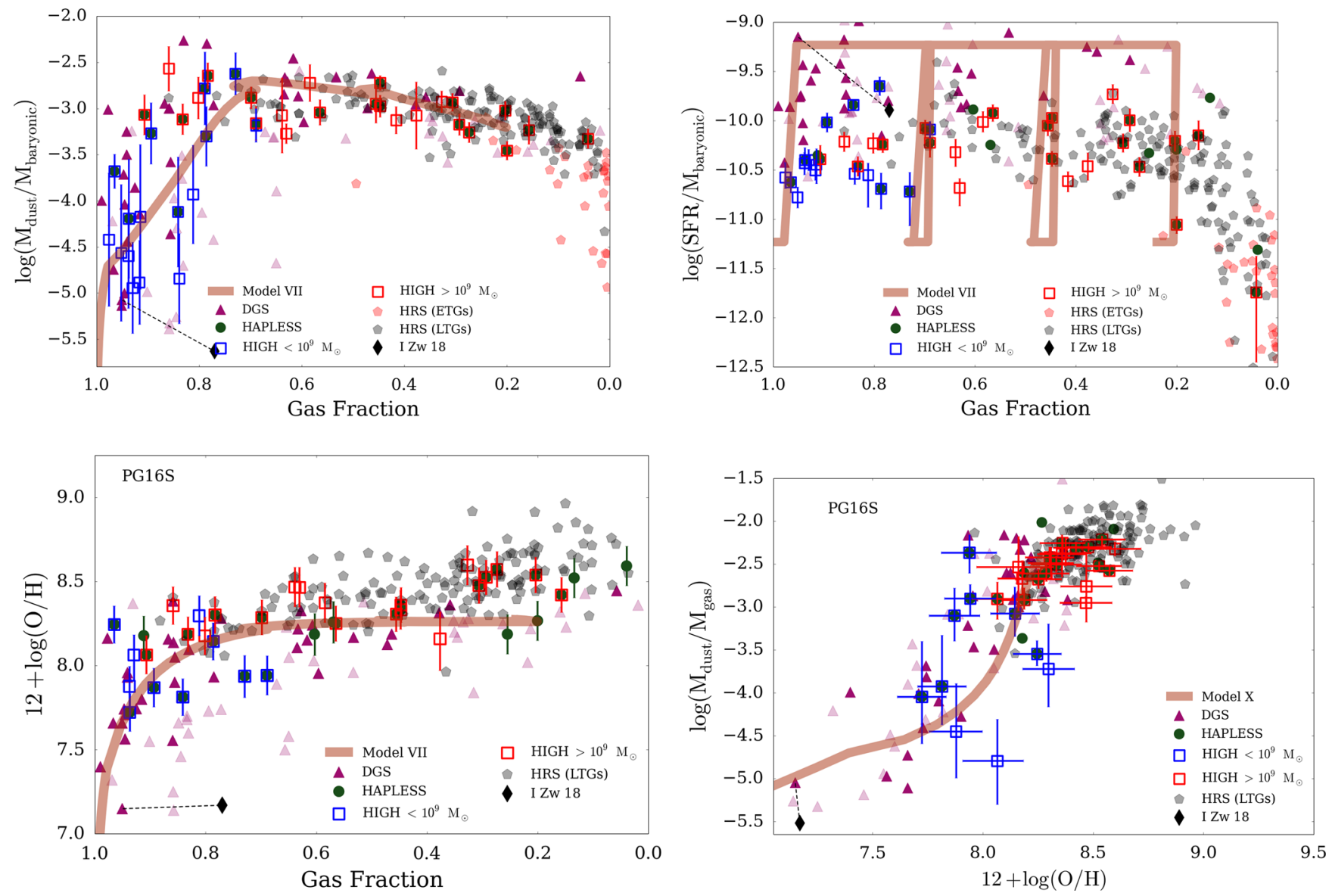

Figure C1. Top: $M_{\mathrm{d}} / M_{\text {bary }}$ and SFR/ $M_{\text {bary }}$ evolution with gas fraction using a bursty SFH (Model VII). As shown in Zhukovska (2014), the DGS sample (purple triangles and transparent triangles are the same galaxies, yet with properties from Zhukovska 2014) can be explained with a model undergoing many bursts of SF (brown line). Bottom: the metallicity variation is compared with gas fraction (left) and $M_{\mathrm{d}} / M_{\mathrm{HI}}$ (right). As shown in Feldmann (2015), the observed metallicity of the DGS galaxies can be explained by a chemical evolution model that incorporates strong inflows and outflows of gas (Model VII).

This paper has been typeset from a $\mathrm{T}_{\mathrm{E}} \mathrm{X} / \mathrm{LT} \mathrm{E} \mathrm{X}$ file prepared by the author. 